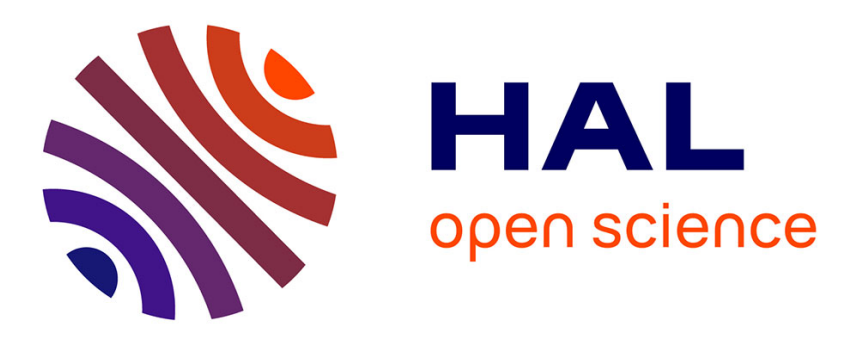

\title{
Altered subcutaneous adipose tissue parameters after switching ART-controlled HIV+ patients to raltegravir/maraviroc
}

Jean-Philippe Bastard, Véronique Pelloux, Rohia Alili, Soraya Fellahi, Judith Aron-Wisnewsky, Emilie Capel, Bruno Fève, Lambert Assoumou, Edi Prifti, Christine Katlama, et al.

\section{To cite this version:}

Jean-Philippe Bastard, Véronique Pelloux, Rohia Alili, Soraya Fellahi, Judith Aron-Wisnewsky, et al.. Altered subcutaneous adipose tissue parameters after switching ART-controlled HIV+ patients to raltegravir/maraviroc. AIDS. Official journal of the international AIDS Society, 2021, Publish Ahead of Print, 10.1097/QAD.0000000000002900 . hal-03200113

\section{HAL Id: hal-03200113 \\ https://hal.sorbonne-universite.fr/hal-03200113}

Submitted on 16 Apr 2021

HAL is a multi-disciplinary open access archive for the deposit and dissemination of scientific research documents, whether they are published or not. The documents may come from teaching and research institutions in France or abroad, or from public or private research centers.
L'archive ouverte pluridisciplinaire HAL, est destinée au dépôt et à la diffusion de documents scientifiques de niveau recherche, publiés ou non, émanant des établissements d'enseignement et de recherche français ou étrangers, des laboratoires publics ou privés. 
Altered subcutaneous adipose tissue parameters after switching ART-controlled HIV+ patients to raltegravir/maraviroc

Jean-Philippe BASTARD ${ }^{1,2}$, Véronique PELLOUX ${ }^{3,4}$, Rohia ALILI ${ }^{3,4}$, Soraya FELLAHI ${ }^{1}$, Judith ARON-WISNEWSKY ${ }^{3,4}$, Emilie CAPEL ${ }^{1}$, Bruno FÈVE ${ }^{1,5}$, Lambert ASSOUMOU ${ }^{6}$, Edi PRIFTI ${ }^{7}$, Christine KATLAMA ${ }^{6,8}$, Karine CLÉMENT ${ }^{3,4}$, Jacqueline CAPEAU ${ }^{1}$

${ }^{1}$ Sorbonne Université, Inserm, Faculty of Medicine, Centre de Recherche Saint-Antoine (CRSA), ICAN, Paris, France

${ }^{2}$ Department of Biochemistry-Pharmacology-Molecular Biology, APHP, Henri-Mondor Hospital, Université Paris Est Créteil, France

${ }^{3}$ Sorbonne Université, Inserm, Faculty of Medicine, Nutrition and obesities: systemic approaches (NutriOmics), Paris, France

${ }^{4}$ Assistance Publique Hôpitaux de Paris, Sorbonne Université, Nutrition department, Pitié-Salpétrière hospital, CRNH Ile-de-France, GH APHP-Sorbonne Université, Paris, France

${ }^{5}$ Department of Endocrinology, CRMR Prisis, Saint-Antoine Hospital, GH APHP-Sorbonne Université, Paris France

${ }^{6}$ Sorbonne Université, Inserm, Faculty of Medicine, Institut Pierre Louis d'Epidémiologie et de Santé Publique (IPLESP), Paris, France

${ }^{7} I R D$, Sorbonne University, UMMISCO, Bondy, France

${ }^{8}$ Department of Infectious Diseases, Pitié-Salpétrière hospital, GH APHP-Sorbonne Université, Paris France 
Short title: fat alterations induced by RAL/MVC

Corresponding author and author to whom requests for reprints should be made:

Pr Jacqueline Capeau

Faculty of Medicine Sorbonne University, 27 rue Chaligny 75012 Paris France

e-mail: jacqueline.capeau@inserm.fr

\section{Conflict of interest and source of funding}

BF has received personal fees for lectures from Amgen, Sanofi, Lilly, Astra-Zeneca, Novo Nordisk

JC has received grants from ViiV healthcare and MSD, and personal fees for lectures from ViiV healthcare, MSD, Gilead, Janssen.

For the remaining authors none were declared

The ANRS 157 ROCnRAL study was sponsored and funded by the French National Institute for Health and Medical Research (INSERM)-French National Agency for Research on AIDS and Viral Hepatitis (ANRS); ViiV Healthcare (France) and Merck Sharp \& Dohme (France) provided maraviroc and raltegravir, respectively, for this trial.

This study received a grant from ANRS. Support was also obtained by RHU CARMMA grant (ANR-15-RHUS-0003), the French National Agency of Research (ANR-Captor project), and ANR-10-IAHU-05 (IHU-ICAN) and FORCE (as part of F-Crin), as well as the Fondation pour la Recherche Médicale (FRM DEQ20120323701 and EQU201903007868). 


\begin{abstract}
Objective: To evaluate the effect on anthropometric, metabolic and adipose tissue parameters of switching ART-controlled persons living with HIV (PLWH) from a proteaseinhibitor regimen to raltegravir/maraviroc.
\end{abstract}

Design: Substudy of ROCnRAL-ANRS157 with investigation of subcutaneous abdominal adipose tissue (SCAT) biopsy at inclusion and study end.

Methods: We performed lipoaspiration of paired SCAT samples, histology on fresh/fixed samples and examined the transcriptomic profile analyzed using Illumina microarrays after RNA extraction. Statistical analyses used Wilcoxon-paired test.

Results: The patients $(n=8)$ were mainly male (7/8), aged (mean \pm SEM) $54.9 \pm 1.2$ years, BMI $26.1 \pm 1.2 \mathrm{~kg} / \mathrm{m}^{2}, \mathrm{CD} 4: 699 \pm 56$ cells $/ \mathrm{mm}^{3}$, all viral load $(\mathrm{VL})<50$ copies $/ \mathrm{mL}$. After a follow-up of $6 \pm 0.5$ months, all PLWH remained with $\mathrm{VL}<50$ copies $/ \mathrm{mL}$. BMI, trunk and limb fat amounts were unchanged yet systemic insulin resistance increased. Adipose tissue histology was unchanged except for borderline increased adipocyte diameter $(P=0.1)$. Amongst the 16,094 RNA transcripts, 458 genes were up-regulated and 244 down-regulated. Analyses of the KEGG and GO databases, evaluating modifications in the main functional pathways, revealed that genes related to immune recognition/function were less expressed as were genes encoding T-cell receptor and receptor signaling pathways. The gene expression profiles indicated decreased 
inflammation but genes involved in adipogenesis and insulin resistance were overexpressed.

Conclusion: After 6 months of raltegravir/maraviroc, adipogenesis-related gene profile was enhanced in SCAT, in agreement with a tendency for increased adipocyte size. Enhanced SCAT insulin resistance-related profile was concordant with higher systemic insulin resistance. However, immune activation/inflammation profile was globally lowered. We propose that raltegravir/maraviroc might favor SCAT gain but reduce inflammation/immune activation.

\section{Key words}

Subcutaneous adipose tissue, raltegravir, maraviroc, transcriptomics, histology, adipogenesis, inflammation/immune activation, insulin resistance 


\section{Introduction}

Integrase strand transfer inhibitors (INSTI) are currently a highly recommended option in ART-naïve and also ART-experienced people living with HIV(PLWH) given their high and rapid efficacy and safe metabolic profile. However, some INSTIs (mainly dolutegravir and bictegravir but also raltegravir) have been recently associated with a global weight and fat mass gain ${ }^{[1]}$. Even if the mechanisms remain elusive, we have previously shown that some INSTIs induced adipocyte hypertrophy, adipose tissue (AT) fibrosis and insulin resistance $^{[2]}$. However, in this previous work evaluating PLWH with severe obesity, we could only cross-compare AT samples issued from INSTI-treated or INSTI-untreated PLWH. The ROCnRAL study (Clinical-Trials.gov: NCT1420523), evaluated the efficacy of a dual therapy associating an INSTI, raltegravir, to the CCR5 inhibitor, maraviroc, in patients suppressed on a protease-inhibitor (PI)-containing regimen but was prematurely stopped due to insufficient virologic control ${ }^{[3]}$. Herein, we analyzed SCAT individual variation before and 6 months after raltegravir/maraviroc initiation.

Increased weight/fat has been reported in PLWH who received some PI-based treatment ${ }^{[1]}$. Due to PI-related increased risk of cardiometabolic diseases, aging PLWH have been often switched to an INSTI-based therapy due to the lipid-lowering effect of INSTI $^{[4-6]}$. However, INSTI-related weight/fat gain also raises concern regarding increased cardiometabolic risk. This risk has not been yet clearly shown for cardiovascular diseases. Regarding insulin resistance and diabetes, results are discrepant $^{[4,7]}$. 
Otherwise, maraviroc has been associated with changes in immune profile and a favorable effect on cardiovascular parameters in $\mathrm{PLWH}^{[8,9]}$. Moreover, maraviroc may exert anti-fibrotic effects on the liver ${ }^{[10,11]}$.

We performed a careful investigation of patients from the ROCnRAL study and examined the individual evolution of anthropometric, metabolic, inflammatory and AT parameters six months after initiation of the dual raltegravir/maraviroc therapy.

\section{Patients and methods}

\section{Patients}

Seventeen patients were enrolled into the ROCnRAL fat sub-study prior to study discontinuation. Eight had subcutaneous-AT (SCAT) biopsies both at initiation and end: they were aged (mean \pm SEM) $54.9 \pm 1.2$ years at inclusion, $87 \%$ male, $87 \%$ Caucasian, $13 \%$ African, CD4 $699 \pm 56$ cells $/ \mathrm{mm}^{3}, \mathrm{VL}<50$ copies/mL. They received raltegravir/maraviroc for a mean duration of $6 \pm 0.5$ months.

\section{Systemic markers}

Adiponectin, leptin and inflammatory markers (hsCRP, hslL-6, sCD14, sCD163) were measured on stored serum samples by immunonephelometry or ELISA ${ }^{[12]}$. Insulin resistance was measured by the quantitative insulin sensitivity check index (QUICKI) = $1 /\left[\log \left(G_{b}\right)+\log \left(I_{b}\right)\right]$ where $G_{b}$ is fasting plasma glucose (milligrams per deciliter), and $I_{b}$ is fasting plasma insulin (microunits per milliliter). 


\section{Fat collection and histology}

Subcutaneous-AT(SCAT) samples were collected by a single operator (JPB) by needle lipoaspiration from the periumbilical area under local anesthesia.

Histology was analyzed on fresh tissue to evaluate adipocyte size/volume and on fixed tissue to evaluate fibrosis by Sirius Red staining (scored using arbitrary units $0-3)^{[13]}$.

\section{mRNA extraction and transcriptomics}

Total RNA was extracted using a RNeasy total-RNA minikit (QIAGEN, Verlo, The Netherlands), quality/concentration assessed (Agilent-2100 bioanalyzer, AgilentTechnologies, Santa-Clara, USA) and biotin-labeled complementary-RNA obtained (Ambion, Thermo-Fischer-Scientific, Waltham, USA). Hybridization processes used Illumina human HT-12 version-4.0 Expression BeadChips (Illumina-Inc, San-Diego, USA). Probes were detected using an Illumina-BeadArray Reader ${ }^{[14]}$.

Raw data were extracted using the numerical results (Illumina Genome-Studio 2011.1 software). The group comparisons used Student's $t$ test. To estimate the false-discoveryrate, we filtered the resulting $P$ values at 5\% and used the Benjamini-Hochberg procedure $^{[14]}$. In the 16 paired samples, $80 \%$ of genes had valid expression. The Significance Analysis of Microarrays (SAM), shown as a switch R plot, indicated the genes significantly up- and down-regulated after the switch (suppl Figure 1). An automated annotation procedure of the differentially expressed genes used the KEGG (Kyoto Encyclopedia of Genes and Genomes) or GO (Gene Ontology) annotation databases. Functional analyses used the FunNet package. 


\section{Statistics}

The paired results were compared using Wilcoxon-test (significant $P<5 \%$, Prism and Statview software). Transcriptomics results were adjusted for multiple testing (significant $\mathrm{q}<5 \%$, SAM package).

\section{Results}

All patients remained controlled $(\mathrm{VL}<50$ copies $/ \mathrm{mL}$ ) with unmodified $\mathrm{CD} 4$ and $\mathrm{CD} 8$ levels (Table 1). BMI was unchanged after 6 months, as waist circumference, waist-to-hip ratio, trunk and limb fat amounts. However, hip circumference increased from 94.9 to 99.1 $\mathrm{cm}(P=0.03)$. Regarding metabolic parameters, insulin increased (10.7 to $21.5 \mathrm{mU} / \mathrm{L}$, $\mathrm{p}<0.05)$. To evaluate insulin resistance, we used QUICKI, better correlated to the gold standard euglycemic hyper-insulinemic clamp IS value than HOMA-IR ${ }^{[15]}$ : QUICKY decreased from 0.342 to $0.315(P<0.05)$, in favor of increased insulin resistance. The level of adiponectin and inflammatory/immune activation markers (hsCRP, hslL-6, sCD14, sCD163) was unmodified but leptin tended to increase ( $P=0.08$, Table 1$)$.

Adipose tissue histology revealed that adipocyte diameter and volume increased yet not reaching significance $(P=0.10)$. The level of parenchyma fibrosis remained unchanged.

Transcriptomic analysis performed in the 16 paired samples identified 16,094 expressed RNA transcripts: 244 genes were down-regulated with a fold-change of 0.66 to 0.9 and 458 genes up-regulated with a fold-change of 10.6 to 1.1 (supplemental Tables 1 and 2). 
Using the GO database, the gene network (Fig 1 ) revealed globally increased DNA replication and transcription, suggesting AT remodeling. Importantly, T-cell receptor signaling pathway was down-regulated as were pathways related to viral infection. Using the KEGG annotation database (Suppl Fig 2), the most enriched function was ubiquitin-mediated proteolysis (19.4\%) and the main impoverished function $(29.7 \%)$ was related to ribosomes involved in protein synthesis, indicating major switch-induced protein remodeling. Functions related to immune cell recognition and immune-related diseases were down-regulated.

Among the individual down- and up-regulated genes, we selected those with the larger amplitude of variation (less than $\times 0.8$ or more than $\times 3$ ). Two main categories were affected: i) genes expressed in immune cells and involved into T-cell and macrophage activation, and ii) genes involved into adipogenesis and insulin sensitivity in adipocytes. Among down-regulated immune-related genes, several encode proteins from the TCR superfamily: CD247 x0.7, TNFRSF4 (tumor-necrosis-factor receptor superfamily member-4) x0.7, CD3D (CD3 molecule $\delta$ in the CD3-TCR complex) x0.7. Other genes encode proteins expressed in T-cells: IL7R x0.7 and GIMAP4 (GTPase, IMAP-familymember-4) x0.7 in the IL7R pathway, GZMB (granzyme B) x0.7, CD58 x0.8, SEMA4D (semaphoring-4D) x0.8, in favor of a decreased level of T-lymphocytes within AT.

Several immune-related genes were upregulated as genes expressed in macrophages favoring the $M 2$ versus $M 1$ phenotype: DAB2/CD11b x4.7, FOXO3 (forkhead-box-O3) x3.9, TCF4 (transcription factor-4) x3.6, CREB1 (CAMP-responsive-element-bindingprotein-1) $\times 3.5$, IL10 x3.1. A number of genes were involved into decreased T- 
lymphocyte immune activation: MAGT1 (magnesium transporter-1) x5.3, DAB2/CD11b x4.7, IL17RD (IL17 receptor-D) x4.0. Also, genes encoding proteins inhibiting NFKB and decreasing inflammation were up-regulated: CDKN2AIPNL (CDKN2A-interactingprotein-N-terminal-like) x7.7, DUSP19 (dual-specificity phosphatase-19) x5.6, KLF6 (Kruppel-like factor-6) x5.5, TRIM3 (tripartite-motif-containing-13) x3.3, while few genes were positively involved into inflammation as SEMA3E (semaphoring-3E) x4.9. Taken as a whole, the gene expression profile suggested decreased T-lymphocytes number/activation and a shift to a M2 macrophage phenotype together with increased expression of anti-inflammatory genes.

Otherwise, up-regulated expression was observed for genes involved into adipogenesis: KLF6 x5.5, QRFRR (polyglutamated RF-amide peptide receptor) x5.5, CREB1 x5.0, FOXO3 x3.9, TRIM13 x3.3, DLC1 (deleted-in-liver-cancer-1) x3.3, IL10 x3.1.

Also, several genes in the insulin signaling pathways involved into increased insulin resistance were up-regulated: SEMA3E x4.9, PIP5K2B (phosphatidylinositol-5phosphate-4-kinase, typelI $\beta$ ) x3.7, IL10 ×3.1, while LEPROT (leptin-receptor-overlappingtranscript) $\times 5.4$ is related to insulin signaling.

\section{Discussion}

We report here that ART-suppressed PLWH switched to raltegravir/maraviroc presented metabolic and adipose tissue modifications over six months. While BMI and fat mass did not change, hip circumference increased indicating fat redistribution. Likewise, insulin resistance, evaluated by fasting glycemia/insulin levels, increased. SCAT exhibited enhanced expression of genes associated with adipogenesis and insulin resistance. 
Otherwise, expression of genes related to T-lymphocytes was reduced and the gene profile suggested a shift towards decreased AT inflammation and a M2 antiinflammatory macrophage phenotype.

INSTI have been recently associated with a global fat mass gain. We previously reported increased adipogenesis induced by dolutegravir and raltegravir in vitro together with adipocyte hypertrophy in SCAT and visceral AT samples issued from INSTI-treated PLWH and macaques ${ }^{[2]}$. Our present results are in good accordance. The fact that the two main categories of modified genes are involved into protein degradation/synthesis enlightens the marked effect of ART versus HIV on fat. Indeed, only switching ART regimen, without CD4 or VL change, deeply affected AT. Previous in vitro studies indicated that maraviroc was neutral regarding adipocyte differentiation ${ }^{[16]}$. Thus, increased adipogenesis, suggested by the gene expression profile, and borderline increased adipocyte size might result from the effect of raltegravir.

Regarding inflammation, in accordance with our present results, we observed in SCAT samples from obese PLWH the absence of inflammation (macrophage infiltration with crown-like structures) in samples issued from INSTI-treated patients by contrast to nonINSTI PLWH and HIV-negative controls ${ }^{[17]}$. Interestingly, increased weight in INSTItreated PLWH was associated with decreased VAT density suggesting a lower inflammation level[18]. Therefore, INSTI could reduce AT inflammation and immune activation. Maraviroc was previously shown to decrease inflammatory cytokine expression in cultured adipocytes ${ }^{[16]}$ and macrophage recruitment in AT induced by a 
high-fat diet in mice ${ }^{[19]}$. Thus, both raltegravir and maraviroc could explain the decreased expression of immune and inflammation-related genes.

Switching to raltegravir/maraviroc was associated with surrogate markers of systemic insulin resistance, probably ART-related. Indeed, BMI remained unmodified and increased hip circumference is generally associated with a favorable metabolic profile. Accordingly, the expression of genes involved in insulin resistance was enhanced in agreement with in vitro studies using raltegravir ${ }^{[2]}$. Since maraviroc has not been reported to affect insulin sensitivity in PLWH and in endothelial cells ${ }^{[20]}$, it is reasonable to hypothesize that the gene profile favoring insulin resistance is related to raltegravir. Clinical studies report discrepant data. We recently reported increased insulin resistance in patients switching from a PI-based regimen to raltegravir-etravirine ${ }^{[21]}$ while, in a similar context, authors concluded that raltegravir increased insulin sensitivity ${ }^{[4]}$.

AT fibrosis adversely impacts metabolic parameters ${ }^{[22]}$. We did not observe any modification in the level of global fibrosis in SCAT and only a few genes which expression was modified were directly related to collagen remodeling. We previously reported increased fibrosis in SCAT of INSTI-treated severely obese PLWH ${ }^{[2]}$. However, maraviroc reduces fibrosis in the liver, and is now evaluated in that setting in clinical trials in PLWH with non-alcoholic-fatty-liver disease ${ }^{[10,11]}$. It might also be anti-fibrotic on AT. Possibly, treatment with both raltegravir and maraviroc could be globally neutral regarding AT fibrosis. 
Circulating levels of adipokines and cytokines remained unchanged but our study lacked potency. Clinical trials using raltegravir or dolutegravir report, or not, modification in the level of these markers ${ }^{[4,5,23]}$. Likewise, maraviroc markedly improved atherosclerotic progression in HIV-suppressed PLWH at high cardiovascular risk ${ }^{[8]}$, but the beneficial effect on the arterial wall was local and not associated with decreased inflammatory systemic markers.

Our study has limitations. Due to premature discontinuation, a small number of patients was enrolled. However, patients monitoring was careful with reliable AT samples withdrawn before and 6 months after the switch, allowing individual comparison. We could not verify the individual level of gene expression nor the change in protein expression and therefore our propositions regarding gene expression variations remain hypothetical. We cannot address the effect of raltegravir versus maraviroc. As well, we cannot analyze the effect of each ART molecules received before switching. Insulin resistance was evaluated by using the surrogate QUICKI index based only on fasting glucose and insulin levels but which was shown more accurate than HOMA-IR ${ }^{[15]}$.

In conclusion, in a small group of well-controlled PLWH, we observed that raltegravir/maraviroc markedly altered AT transcriptome suggesting increased adipogenesis, probably latter resulting in fat mass gain. Also, insulin resistance increased stressing for the risk of diabetes, requiring a careful follow-up. However, we observed a striking reduction in the expression of immune/inflammation-related genes. These 
preliminary lead to interesting hypotheses which need to be confirmed in larger studies as they may impact the use of INSTI in some populations.

\section{Legend of figures}

Figure 1: Network analysis with the GO biological process annotation of genes with altered expression 6 months after switch to raltegravir/maraviroc

Green: decreased expression

Red: increased expression

Supplemental Figure 1: Significance Analysis of Microarrays (SAM) analysis of comparative adipose tissue gene expression after versus before the switch to raltegravir/maraviroc

Supplemental Figure 2: Functional analysis by using the KEGG database of adipose tissue genes up- or down-regulated after switch to raltegravir/maraviroc Green: decreased expression

Red: increased expression 


\section{Acknowledgements}

We thank the clinical research department from ANRS for their support regarding the management of the ROCnRAL study. We thank Ginette Marlin for evaluation of the adipokines and inflammatory markers.

JPB performed all the lipoaspirations.

VP, RA, JPB, SF, EC and EP have performed all the assays presented in this paper and participated to their analysis.

LA has monitored the ROCnRAL study

VP, LA, JPB and JC have performed the analysis of the data

CK has participated to the study as the PI of the ROCnRAL study

JPB, JC, CK and KC have written the grant proposition

$\mathrm{JC}$ has written the first draft, $\mathrm{BF}, \mathrm{JAW}, \mathrm{KC}$ and $\mathrm{CK}$ have participated to the redaction and all the authors have participated to the correction of the paper 


\section{References}

1. Koethe JR, Lagathu C, Lake JE, Domingo P, Calmy A, Falutz J, et al. HIV and antiretroviral therapy-related fat alterations. Nat Rev Dis Primers 2020; 6(1):48.

2. Gorwood J, Bourgeois C, Pourcher V, Pourcher G, Charlotte F, Mantecon M, et al. The integrase inhibitors dolutegravir and raltegravir exert pro-adipogenic and profibrotic effects and induce insulin resistance in human/simian adipose tissue and human adipocytes. Clin Infect Dis 2020.

3. Katlama C, Assoumou L, Valantin MA, Soulie C, Duvivier C, Chablais L, et al. Maraviroc plus raltegravir failed to maintain virological suppression in HIV-infected patients with lipohypertrophy: results from the ROCnRAL ANRS 157 study. J Antimicrob Chemother $2014 ; 69(6): 1648-1652$.

4. Martinez E, D'Albuquerque PM, Llibre JM, Gutierrez F, Podzamczer D, Antela A, et al. Changes in cardiovascular biomarkers in HIV-infected patients switching from ritonavir-boosted protease inhibitors to raltegravir. AIDS 2012; 26(18):2315-2326.

5. Katlama C, Assoumou L, Valantin MA, Soulie C, Martinez E, Beniguel L, et al. Dual therapy combining raltegravir with etravirine maintains a high level of viral suppression over 96 weeks in long-term experienced HIV-infected individuals over 45 years on a PI-based regimen: results from the Phase II ANRS 163 ETRAL study. J Antimicrob Chemother 2019; 74(9):2742-2751.

6. Gatell JM, Assoumou L, Moyle G, Waters L, Johnson M, Domingo P, et al. Immediate Versus Deferred Switching From a Boosted Protease Inhibitor-based Regimen to a Dolutegravir-based Regimen in Virologically Suppressed Patients With High 
Cardiovascular Risk or Age >/=50 Years: Final 96-Week Results of the NEAT022 Study. Clin Infect Dis 2019; 68(4):597-606.

7. Assoumou L, di Clemente N, Fellahi S, Beniguel L, Bastard JP, Feve B, et al. Impact of the reproductive/hormonal status on weight, fat and insulin resistance in HIV-infected women switching from a PI regimen to dual raltegravir-etravirine therapy: results from the ANRS163-ETRAL trial at 48 and 96 weeks. HIV Med 2019; 20(SI 9):132.

8. Francisci D, Pirro M, Schiaroli E, Mannarino MR, Cipriani S, Bianconi V, et al. Maraviroc Intensification Modulates Atherosclerotic Progression in HIV-Suppressed Patients at High Cardiovascular Risk. A Randomized, Crossover Pilot Study. Open Forum Infect Dis 2019; 6(4):ofz112.

9. Piconi S, Pocaterra D, Rainone V, Cossu M, Masetti M, Rizzardini G, et al. Maraviroc Reduces Arterial Stiffness in PI-Treated HIV-infected Patients. Sci Rep 2016; 6:28853. 10. Gonzalez EO, Boix V, Deltoro MG, Aldeguer JL, Portilla J, Montero M, et al. The effects of Maraviroc on liver fibrosis in HIV/HCV co-infected patients. J Int AIDS Soc 2014; 17(4 Suppl 3):19643.

11. Bradshaw D, Gilleece Y, Verma S, Abramowicz I, Bremner S, Perry N. Protocol for a phase IV, open-label feasibility study investigating non-invasive markers of hepatic fibrosis in people living with HIV-1 and non-alcoholic fatty liver disease randomised to receiving optimised background therapy (OBT) plus maraviroc or OBT alone. BMJ Open 2020; 10(7):e035596.

12. Bastard JP, Fellahi S, Couffignal C, Raffi F, Gras G, Hardel L, et al. Increased systemic immune activation and inflammatory profile of long-term HIV-infected ART-controlled 
patients is related to personal factors, but not to markers of HIV infection severity. $J$ Antimicrob Chemother 2015; 70(6):1816-1824.

13. Gorwood J, Bourgeois C, Mantecon M, Atlan M, Pourcher V, Pourcher G, et al. Impact of HIV/simian immunodeficiency virus infection and viral proteins on adipose tissue fibrosis and adipogenesis. AIDS 2019; 33(6):953-964.

14. Lacroix D, Moutel S, Coupaye M, Huvenne H, Faucher P, Pelloux V, et al. Metabolic and adipose tissue signatures in adults with Prader-Willi syndrome: a model of extreme adiposity. J Clin Endocrinol Metab 2015; 100(3):850-859.

15. Rabasa-Lhoret R, Bastard JP, Jan V, Ducluzeau P, Andreelli F, Guerbre F, et al. Modified quantitative insulin sensitivity check index is better correlated to hyperinsulinemic glucose clamp than other fasting-based index of insulin sesnitivity in different insulin-resistant states. J Clin Endocrinol Metab 2003; 88(10):4917-4923.

16. Diaz-Delfin J, Domingo P, Giralt M, Villarroya F. Maraviroc reduces cytokine expression and secretion in human adipose cells without altering adipogenic differentiation. Cytokine 2013; 61(3):808-815.

17. Pourcher V, Dutoit Y, Capeau J, Boccara F, Soulie C, Ndoadoumgue A, et al. Characteristics of HIV+ and HIV- patients undergoing bariatric surgery: ObeVIH study. In: virtual CROI scientific spotlight-TM ID1717. virtual; 2021.

18. Guaraldi G, Draisci J, Milic J, Carli F, Besutti G, Bassoli C, et al. Fat distribution and ensity in people living with HIV with $\geq 5 \%$ weight gain. J Intern AIDS Soc 2020; 23(S7):E25616.

19. Perez-Matute P, Pichel JG, Iniguez M, Recio-Fernandez E, Perez-Martinez L, Torrens $\mathrm{R}$, et al. Maraviroc ameliorates the increased adipose tissue macrophage recruitment 
induced by a high-fat diet in a mouse model of obesity. Antivir Ther 2017; 22(2):163168.

20. Auclair M, Guenantin AC, Fellahi S, Garcia M, Capeau J. HIV antiretroviral drugs, dolutegravir, maraviroc and ritonavir-boosted atazanavir use different pathways to affect inflammation, senescence and insulin sensitivity in human coronary endothelial cells. PLoS One 2020; 15(1):e0226924.

21. Assoumou L, Racine C, Fellahi S, Lamaziere A, Farabos D, Beniguel L, et al. Fat gain differs by sex and hormonal status in persons living with suppressed HIV switched to raltegravir/etravirine. AIDS 2020; 34(12):1859-1862.

22. Divoux A, Tordjman J, Lacasa D, Veyrie N, Hugol D, Aissat A, et al. Fibrosis in human adipose tissue: composition, distribution, and link with lipid metabolism and fat mass loss. Diabetes 2010; 59(11):2817-2825.

23. Martinez E, Assoumou L, Moyle G, Waters L, Johnson M, Domingo P, et al. 48-week changes in biomarkers in subjects with high cardiovascular risk boosted switching from ritonavir-protease inhibitors to dolutegravir: the NEAT022 study. Journal of the International AIDS Society 2018; 21(S8):e25187. 
Table 1

Characteristics of PLWH included in the ROCnRAL sub-study with paired adipose tissue biopsies: clinical and metabolic parameters, adipokines and inflammatory markers, adipose tissue parameters.

The results are expressed as mean (SEM). The differences between TO (inclusion) and Tend was calculated by using the Wilcoxon-paired test.

\begin{tabular}{|l|c|c|c|}
\hline & T0 & Tend & $P$ \\
\hline CD4 cells/mm ${ }^{3}$ & $699(56)$ & $612(23)$ & 0.219 \\
\hline CD8 cells/mm ${ }^{3}$ & $786(100)$ & $820(111)$ & 0.359 \\
\hline BMI kg/m & $26.1(1.2)$ & $26.4(1.3)$ & 0.578 \\
\hline WC cm & $94.6(2.2)$ & $97.9(4.0)$ & 0.250 \\
\hline HC cm & $94.9(1.8)$ & $99.1(2.1)$ & 0.031 \\
\hline WHR & $1.00(0.02)$ & $0.98(0.02)$ & 0.250 \\
\hline Trunk fat kg & $13.2(1.4)$ & $13.6(1.8)$ & 0.562 \\
\hline Limb fat kg & $8.0(1.2)$ & $8.1(1.2)$ & 0.312 \\
\hline Glycemia mmol/L & $5.1(0.2)$ & $5.5(0.25)$ & 0.250 \\
\hline Triglycerides mmol/L & $2.0(0.9)$ & $1.3(0.2)$ & 0.437 \\
\hline HDL-cholesterol mmol/L & $1.35(0.14)$ & $1.5(0.12)$ & 0.219 \\
\hline LDL-cholesterol mmol/L & $3.3(0.33)$ & $2.8(0.24)$ & 0.219 \\
\hline Insulinemia mU/L & $10.7(1.8)$ & $21.5(10.0)$ & $0.315(0.017)$ \\
\hline QUICKI & $0.342(0.011)$ & & \\
\hline & & & \\
\hline & & & \\
\hline
\end{tabular}




\begin{tabular}{|l|c|c|c|}
\hline hsCRP mg/L & $2.61(1.06)$ & $3.8(1.7)$ & 0.578 \\
\hline hslL-6 pg/L & $4.5(2.5)$ & $2.7(0.9)$ & 0.937 \\
\hline Adiponectin mg/L & $3.9(0.8)$ & $4.4(1.1)$ & 0.937 \\
\hline Leptin $\mu \mathrm{g} / \mathrm{L}$ & $12.4(4.4)$ & $18.2(5.1)$ & 0.078 \\
\hline sCD14 ng/ml & $1583(71)$ & $1471(101)$ & 0.219 \\
\hline sCD163 ng/ml & $699(103)$ & $790(151)$ & 0.467 \\
\hline Adipocyte diameter $\mu \mathrm{m}$ & $106.9(2.1)$ & $113(4.1)$ & 0.109 \\
\hline Adipocyte volume & $649(38)$ & $776(78)$ & 0.109 \\
x103 $\mu m^{3}$ & & & 0.999 \\
\hline Adipose tissue fibrosis AU & $1.37(0.26)$ & $1.56(0.35)$ & \\
\hline
\end{tabular}

WC: waist circumference, HC: hip circumference, WHR: waist to hip ratio, HDL: high density lipoproteins, LDL: low density lipoproteins, AU: arbitrary unit 


\section{Figure 1}

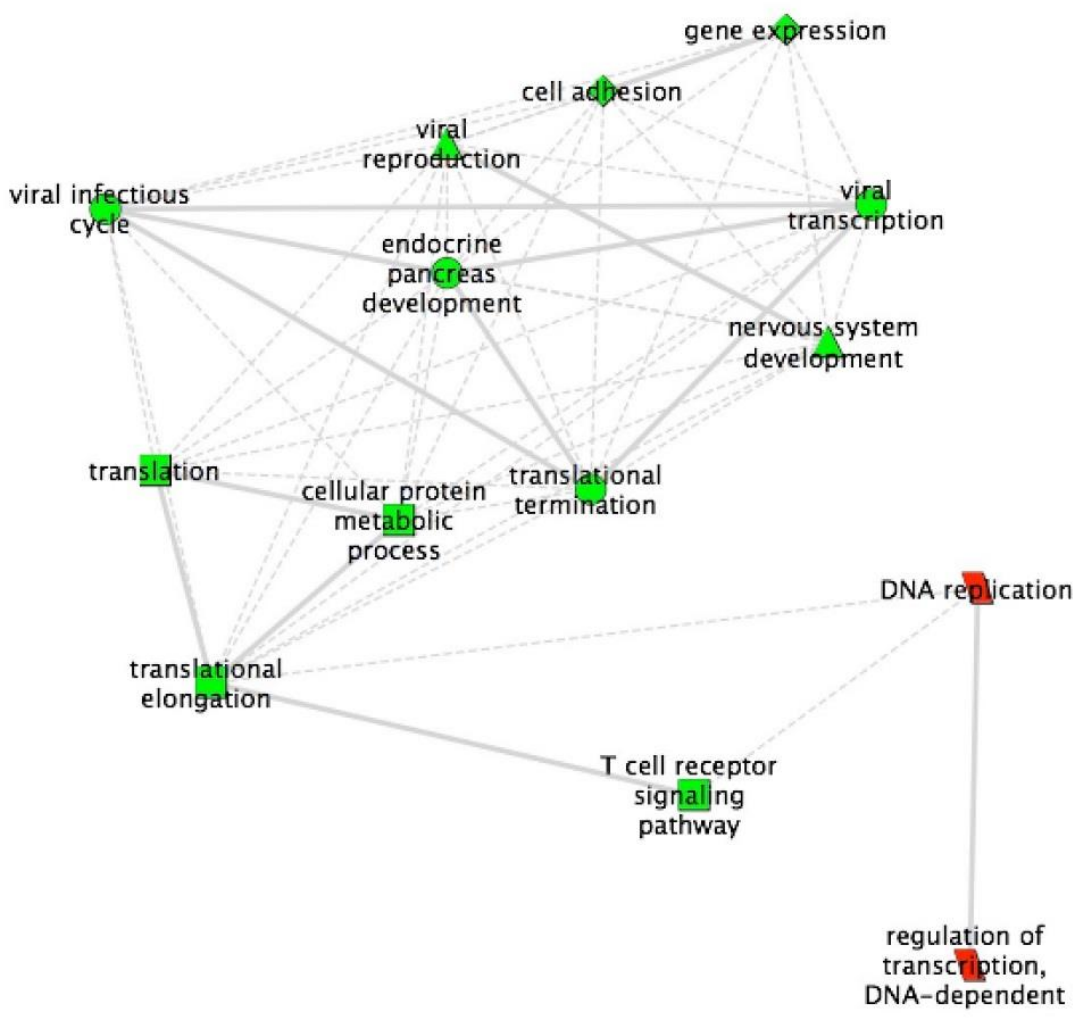

Green: decreased expression

Red: increased expression 


\begin{tabular}{|c|c|c|c|c|c|}
\hline Gene ID & $\underline{\text { ID }}$ & Gene symbc & I Name & Eold C & -value(\% \\
\hline ILMN_16829! & 4818 & NKG7 & natural killer cell group 7 sequence & 0,7 & 1,8141 \\
\hline ILMN_237761 & 919 & CD247 & CD247 molecule & 0,7 & 1,2499 \\
\hline ILMN_21206! & 7102 & TSPAN7 & tetraspanin 7 & 0,7 & 1,8141 \\
\hline ILMN_16768: & 84417 & C2orf40 & chromosome 2 open reading frame 40 & 0,7 & 2,0799 \\
\hline ILMN_21094i & 3002 & GZMB & granzyme B (granzyme 2, cytotoxic T-Iy & 0,7 & 2,0799 \\
\hline ILMN_17695" & 83700 & JAM3 & junctional adhesion molecule 3 & 0,7 & 1,5007 \\
\hline ILMN_17494: & 8502 & PKP4 & plakophilin 4 & 0,7 & 2,0799 \\
\hline ILMN_21122! & 7293 & TNFRSF4 & tumor necrosis factor receptor superfal & 0,7 & 2,6244 \\
\hline ILMN_22090: & 6231 & RPS26 & ribosomal protein S26 & 0,7 & 2,0799 \\
\hline ILMN_16769: & 919 & CD247 & CD247 molecule & 0,7 & 1,5007 \\
\hline ILMN_20862: & 114826 & SMYD4 & SET and MYND domain containing 4 & 0,7 & 2,0799 \\
\hline ILMN_178751 & 8848 & TSC22D1 & TSC22 domain family, member 1 & 0,7 & 1,2499 \\
\hline ILMN_32368! & 57523 & NYNRIN & NYN domain and retroviral integrase $\mathrm{cc}$ & 0,7 & 2,0799 \\
\hline ILMN_24063: & 10616 & RBCK1 & RanBP-type and C3HC4-type zinc finger & 0,7 & 2,0799 \\
\hline ILMN_17707: & 83543 & AlF1L & allograft inflammatory factor 1-like & 0,7 & 2,0799 \\
\hline ILMN_32517: & 79002 & C19orf43 & chromosome 19 open reading frame $4 \equiv$ & 0,7 & 2,6244 \\
\hline ILMN_22614: & 915 & CD3D & CD3d molecule, delta (CD3-TCR comple & 0,7 & 1,2499 \\
\hline ILMN_211231 & 10589 & DRAP1 & DR1-associated protein 1 (negative cof: & 0,7 & 2,6244 \\
\hline ILMN_16723! & 58494 & JAM2 & nesion molecule 2 & 0,7 & 0,0000 \\
\hline ILMN_176961 & 5878 & RAB5C & RAB5C, member RAS oncogene family & 0,7 & 2,6244 \\
\hline ILMN_16913، & 3575 & IL7R & interleukin 7 receptor & 0,7 & 1,8141 \\
\hline ILMN_23162: & 84525 & & & 0,7 & 007 \\
\hline ILMN_17484" & 55303 & GIMAP4 & GTPase, IMAP family member 4 & 0,7 & 2,0799 \\
\hline ILMN_17171| & 3476 & IGBP1 & immunoglobulin (CD79A) binding prote & 0,8 & 2,0799 \\
\hline ILMN_32502: & 29767 & TMOD2 & tropomodulin 2 (neuronal) & 0,8 & 1,8141 \\
\hline ILMN_17816: & 716 & C1S & complement component 1 , s subcompc & 0,8 & 1,8141 \\
\hline ILMN_17761! & 283298 & OLFML1 & olfactomedin-like 1 & 0,8 & 0,8244 \\
\hline ILMN_31889: & 441951 & C20orf199 & ZNFX1 antisense RNA 1 (non-protein co & 0,8 & 1,0331 \\
\hline ILMN_324331 & 343990 & & chromosome 2 open reading frame 55 & 0,8 & 0,0000 \\
\hline ILMN_17319: & 6329 & SCN4A & sodium channel, voltage-gated, type IV & 0,8 & 1,8141 \\
\hline ILMN_16704! & 79171 & RBM42 & RNA binding motif protein 42 & 0,8 & 2,6244 \\
\hline ILMN_217481 & 146894 & CD300LG & CD300 molecule-like family member g & 0,8 & 2,0799 \\
\hline ILMN_178521 & 965 & CD58 & CD58 molecule & 0,8 & 2,0799 \\
\hline ILMN_18057: & 5214 & PFKP & phosphofructokinase, platelet & 0,8 & 2,6244 \\
\hline ILMN_16875: & 10507 & SEMA4D & sema domain, immunoglobulin domain & 0,8 & 1,2499 \\
\hline ILMN_16512i & 255877 & BCL6B & B-cell CLL/lymphoma 6, member B & 0,8 & 2,6244 \\
\hline ILMN_16984| & 3609 & ILF3 & interleukin enhancer binding factor $3, \subseteq$ & 0,8 & 2,0799 \\
\hline ILMN_23882: & 9862 & MED24 & mediator complex subunit 24 & 0,8 & 2,6244 \\
\hline ILMN_18060: & 5720 & PSME1 & proteasome (prosome, macropain) acti & 0,8 & 2,6244 \\
\hline ILMN_23841: & 9289 & GPR56 & G protein-coupled receptor 56 & 0,8 & 1,8141 \\
\hline ILMN_17695، & 54453 & RIN2 & Ras and Rab interactor 2 & 0,8 & 2,0799 \\
\hline ILMN_17545| & 9828 & ARHGEF17 & Rho guanine nucleotide exchange facto & 0,8 & 1,5007 \\
\hline ILMN_16890! & 79673 & ZNF329 & zinc finger protein 329 & 0,8 & 2,6244 \\
\hline ILMN_16582i & 6139 & RPL17 & ribosomal protein L17 & 0,8 & 1,8141 \\
\hline ILMN_32514" & 9804 & TOMM20 & translocase of outer mitochondrial mer & 0,8 & 1,5007 \\
\hline
\end{tabular}




\begin{tabular}{|c|c|c|c|c|c|}
\hline ILMN_21574، & 3122 & HLA-DRA & major histocompatibility complex, class & 0,8 & 2,6244 \\
\hline ILMN_17478! & 6605 & SMARCE1 & SWI/SNF related, matrix associated, act & 0,8 & 2,0799 \\
\hline ILMN_17959: & 116988 & CENTG3 & ArfGAP with GTPase domain, ankyrin re & 0,8 & 2,6244 \\
\hline ILMN_23258: & 915 & CD3D & CD3d molecule, delta (CD3-TCR comple & 0,8 & 2,6244 \\
\hline ILMN_16857: & 1974 & EIF4A2 & eukaryotic translation initiation factor $\angle$ & 0,8 & 1,5007 \\
\hline ILMN_18044: & 57228 & SMAGP & small cell adhesion glycoprotein & 0,8 & 1,5007 \\
\hline ILMN_18134! & 5092 & PCBD1 & pterin-4 alpha-carbinolamine dehydrat & 0,8 & 2,6244 \\
\hline ILMN_165351 & 1901 & EDG1 & sphingosine-1-phosphate receptor 1 & 0,8 & 2,6244 \\
\hline ILMN_17217، & 54707 & ATPBD1B & GPN-loop GTPase 2 & 0,8 & 2,6244 \\
\hline ILMN_32438! & 4695 & NDUFA2 & NADH dehydrogenase (ubiquinone) $1 \mathrm{a}$ & 0,8 & 1,0331 \\
\hline ILMN_16608i & 6050 & $\mathrm{RNH1}$ & ribonuclease/angiogenin inhibitor 1 & 0,8 & 2,6244 \\
\hline ILMN_17303، & 84317 & CCDC115 & coiled-coil domain containing 115 & 0,8 & 2,0799 \\
\hline ILMN_16597| & 689 & BTF3 & basic transcription factor 3 & 0,8 & 1,5007 \\
\hline ILMN_22537: & 7903 & ST8SIA4 & ST8 alpha-N-acetyl-neuraminide alpha- & 0,8 & 1,8141 \\
\hline ILMN_21202: & 10231 & RCAN2 & regulator of calcineurin 2 & 0,8 & 2,6244 \\
\hline ILMN_17433، & 113189 & CHST14 & carbohydrate (N-acetylgalactosamine 4 & 0,8 & 2,6244 \\
\hline ILMN_17592! & 118 & ADD1 & adducin 1 (alpha) & 0,8 & 1,2499 \\
\hline ILMN_16768! & 116835 & HSPA12B & heat shock 70kD protein 12B & 0,8 & 1,8141 \\
\hline ILMN_17690! & 5547 & PRCP & prolylcarboxypeptidase (angiotensinas€ & 0,8 & 2,0799 \\
\hline ILMN_23402! & 5142 & PDE4B & phosphodiesterase 4B, cAMP-specific & 0,8 & 0,0000 \\
\hline ILMN_18007: & 23180 & RFTN1 & raftlin, lipid raft linker 1 & 0,8 & 2,6244 \\
\hline ILMN_23372، & 6210 & RPS15A & ribosomal protein S15a & 0,8 & 1,0331 \\
\hline ILMN_16780" & 9425 & CDYL & chromodomain protein, Y-like & 0,8 & 1,8141 \\
\hline ILMN_16590" & 3111 & HLA-DOA & major histocompatibility complex, class & 0,8 & 1,8141 \\
\hline ILMN_18083! & 9744 & ACAP1 & ArfGAP with coiled-coil, ankyrin repeat & 0,8 & 2,0799 \\
\hline ILMN_16804| & 6748 & SSR4 & signal sequence receptor, delta & 0,8 & 2,6244 \\
\hline ILMN_20796! & 3820 & KLRB1 & killer cell lectin-like receptor subfamily & 0,8 & 1,2499 \\
\hline ILMN_18129| & 116159 & CYYR1 & cysteine/tyrosine-rich 1 & 0,8 & 2,0799 \\
\hline ILMN_17973، & 23048 & FNBP1 & formin binding protein 1 & 0,8 & 2,0799 \\
\hline ILMN_17823i & 5430 & POLR2A & polymerase (RNA) II (DNA directed) pol & 0,8 & 1,5007 \\
\hline ILMN_22050: & 57692 & MAGEE1 & melanoma antigen family $E, 1$ & 0,8 & 2,0799 \\
\hline ILMN_23520! & 9289 & GPR56 & G protein-coupled receptor 56 & 0,8 & 0,0000 \\
\hline ILMN_17917! & 1375 & CPT1B & carnitine palmitoyltransferase 1B (musı & 0,8 & 2,6244 \\
\hline ILMN_16797! & 104 & ADARB1 & adenosine deaminase, RNA-specific, B1 & 0,8 & 2,6244 \\
\hline ILMN_23830! & 6139 & RPL17 & ribosomal protein L17 & 0,8 & 2,0799 \\
\hline ILMN_232511 & 408 & ARRB1 & arrestin, beta 1 & 0,8 & 0,8244 \\
\hline ILMN_18038: & 84518 & CNFN & cornifelin & 0,8 & 2,6244 \\
\hline ILMN_16680! & 90952 & ESAM & endothelial cell adhesion molecule & 0,8 & 2,6244 \\
\hline ILMN_17775: & 3695 & ITGB7 & integrin, beta 7 & 0,8 & 1,5007 \\
\hline ILMN_16657! & 1434 & CSE1L & CSE1 chromosome segregation 1-like (y & 0,8 & 2,6244 \\
\hline ILMN_235231 & 9770 & RASSF2 & Ras association (RalGDS/AF-6) domain 1 & 0,8 & 2,6244 \\
\hline ILMN_18151! & 4628 & MYH10 & myosin, heavy chain 10, non-muscle & 0,8 & 2,6244 \\
\hline ILMN_16772: & 55217 & TMLHE & trimethyllysine hydroxylase, epsilon & 0,8 & 2,0799 \\
\hline ILMN_16519! & 8460 & TPST1 & tyrosylprotein sulfotransferase 1 & 0,8 & 1,2499 \\
\hline ILMN_16779: & 51292 & GMPR2 & guanosine monophosphate reductase 2 & 0,8 & 1,2499 \\
\hline ILMN_21179| & 7570 & ZNF22 & zinc finger protein 22 (KOX 15) & 0,8 & 2,6244 \\
\hline
\end{tabular}




\begin{tabular}{|c|c|c|c|c|c|}
\hline ILMN_17006| & 84282 & RNF135 & ring finger protein 135 & 0,8 & 2,6244 \\
\hline ILMN_17808! & 5583 & PRKCH & protein kinase $C$, eta & 0,8 & 0,7857 \\
\hline ILMN_17754، & 5217 & PFN2 & profilin 2 & 0,8 & 2,6244 \\
\hline ILMN_17754i & 8082 & SSPN & sarcospan & 0,8 & 2,0799 \\
\hline ILMN_32394، & 100128927 & ZBTB42 & zinc finger and BTB domain containing ، & 0,8 & 1,5007 \\
\hline ILMN_17975: & 84269 & CHCHD5 & coiled-coil-helix-coiled-coil-helix domai & 0,8 & 2,6244 \\
\hline ILMN_21916i & 51142 & $\mathrm{CHCHD} 2$ & coiled-coil-helix-coiled-coil-helix domai & 0,8 & 2,0799 \\
\hline ILMN_16940! & 27335 & EIF3K & eukaryotic translation initiation factor & 0,8 & 0,8244 \\
\hline ILMN_17813i & 5239 & PGM5 & phosphoglucomutase 5 & 0,8 & 1,8141 \\
\hline ILMN_17513: & 10616 & RBCK1 & RanBP-type and C3HC4-type zinc finger & 0,8 & 1,8141 \\
\hline ILMN_16557| & 54460 & MRPS21 & mitochondrial ribosomal protein S21 & 0,8 & 1,8141 \\
\hline ILMN_17831، & 6192 & RPS4Y1 & ribosomal protein S4, Y-linked 1 & 0,8 & 1,5007 \\
\hline ILMN_232651 & 834 & CASP1 & caspase 1 , apoptosis-related cysteine $p$ & 0,8 & 2,6244 \\
\hline ILMN_20870| & 54543 & TOMM7 & translocase of outer mitochondrial mer & 0,8 & 2,0799 \\
\hline ILMN_17395! & 78987 & CRELD1 & cysteine-rich with EGF-like domains 1 & 0,8 & 1,2499 \\
\hline ILMN_17095! & 5239 & PGM5 & phosphoglucomutase 5 & 0,8 & 1,8141 \\
\hline ILMN_181131 & 2494 & NR5A2 & nuclear receptor subfamily 5 , group $A$, & 0,8 & 1,8141 \\
\hline ILMN_17761! & 5414 & 38231 & septin 4 & 0,8 & 1,8141 \\
\hline ILMN_17357" & 3764 & KCNJ8 & potassium inwardly-rectifying channel, & 0,8 & 2,0799 \\
\hline ILMN_17558: & 85360 & SYDE1 & synapse defective 1 , Rho GTPase, homc & 0,8 & 2,0799 \\
\hline ILMN_20876! & 79901 & CYBRD1 & cytochrome b reductase 1 & 0,8 & 2,0799 \\
\hline ILMN_23826، & 11043 & MID2 & midline 2 & 0,8 & 2,0799 \\
\hline ILMN_21676: & 4666 & NACA & nascent polypeptide-associated compl€ & 0,8 & 2,0799 \\
\hline ILMN_21545| & 4736 & RPL10A & ribosomal protein L10a & 0,8 & 2,0799 \\
\hline ILMN_16711| & 2701 & GJA4 & gap junction protein, alpha 4, 37kDa & 0,8 & 1,0331 \\
\hline ILMN_23345i & 3183 & HNRNPC & heterogeneous nuclear ribonucleoprotı & 0,8 & 2,0799 \\
\hline ILMN_16568: & 5947 & RBP1 & retinol binding protein 1 , cellular & 0,8 & 2,6244 \\
\hline ILMN_16990: & 8550 & MAPKAPK5 & mitogen-activated protein kinase-activi & 0,8 & 1,0331 \\
\hline ILMN_21399، & 6189 & RPS3A & ribosomal protein S3A & 0,8 & 0,8244 \\
\hline ILMN_17365، & 151473 & SLC16A14 & solute carrier family 16 , member 14 (m & 0,8 & 1,5007 \\
\hline ILMN_17116| & 25824 & PRDX5 & peroxiredoxin 5 & 0,8 & 1,5007 \\
\hline ILMN_22269: & 9766 & KIAA0247 & KIAA0247 & 0,8 & 2,0799 \\
\hline ILMN_23685: & 115290 & FBX017 & F-box protein 17 & 0,8 & 2,0799 \\
\hline ILMN_17115: & 1690 & $\mathrm{COCH}$ & coagulation factor $\mathrm{C}$ homolog, cochlin ( & 0,8 & 1,2499 \\
\hline ILMN_17612! & 2132 & EXT2 & exostosin 2 & 0,8 & 1,5007 \\
\hline ILMN_20730: & 94107 & TMEM203 & transmembrane protein 203 & 0,8 & 2,0799 \\
\hline ILMN_17496: & 8454 & CUL1 & cullin 1 & 0,8 & 1,0331 \\
\hline ILMN_17164i & 65057 & ACD & adrenocortical dysplasia homolog (mou & 0,8 & 1,2499 \\
\hline ILMN_16740| & 54543 & TOMM7 & translocase of outer mitochondrial mer & 0,8 & 1,2499 \\
\hline ILMN_23194: & 689 & BTF3 & basic transcription factor 3 & 0,8 & 2,0799 \\
\hline ILMN_17627، & 6138 & RPL15 & ribosomal protein L15 & 0,8 & 1,5007 \\
\hline ILMN_17651، & 3455 & IFNAR2 & interferon (alpha, beta and omega) rea & 0,8 & 2,6244 \\
\hline ILMN_17394! & 5396 & PRRX1 & paired related homeobox 1 & 0,8 & 2,6244 \\
\hline ILMN_16920! & 4692 & NDN & necdin homolog (mouse) & 0,8 & 2,6244 \\
\hline ILMN_17494: & 64983 & MRPL32 & mitochondrial ribosomal protein L32 & 0,8 & 1,8141 \\
\hline ILMN_18104i & 83871 & RAB34 & RAB34, member RAS oncogene family & 0,8 & 1,2499 \\
\hline
\end{tabular}




\begin{tabular}{|c|c|c|c|c|c|}
\hline ILMN_17306: & 408 & ARRB1 & arrestin, beta 1 & 0,8 & 2,0799 \\
\hline ILMN_18102: & 90423 & ATP6V1E2 & ATPase, $\mathrm{H}+$ transporting, lysosomal 31k & 0,8 & 2,0799 \\
\hline ILMN_21012: & 64407 & RGS18 & regulator of G-protein signaling 18 & 0,8 & 1,5007 \\
\hline ILMN_32453! & 84747 & UNC119B & unc-119 homolog B (C. elegans) & 0,8 & 2,6244 \\
\hline ILMN_18116! & 117246 & FTSJ3 & FtsJ homolog 3 (E. coli) & 0,8 & 2,6244 \\
\hline ILMN_16801! & 55353 & LAPTM4B & Iysosomal protein transmembrane 4 be & 0,8 & 2,0799 \\
\hline ILMN_17611: & 286257 & C9orf142 & chromosome 9 open reading frame 142 & 0,8 & 1,2499 \\
\hline ILMN_17145| & 79026 & AHNAK & AHNAK nucleoprotein & 0,8 & 0,0000 \\
\hline ILMN_16787| & 6993 & DYNLT1 & dynein, light chain, Tctex-type 1 & 0,8 & 2,0799 \\
\hline ILMN_24002: & 6717 & SRI & sorcin & 0,8 & 2,6244 \\
\hline ILMN_17601i & 11043 & MID2 & midline 2 & 0,8 & 1,8141 \\
\hline ILMN_17204 & 5253 & PHF2 & PHD finger protein 2 & 0,8 & 1,8141 \\
\hline ILMN_18031: & 23450 & SF3B3 & splicing factor $3 b$, subunit $3,130 \mathrm{kDa}$ & 0,8 & 1,2499 \\
\hline ILMN_18026! & 83468 & GLT8D2 & glycosyltransferase 8 domain containin & 0,8 & 1,8141 \\
\hline ILMN_208731 & 55113 & XKR8 & XK, Kell blood group complex subunit-rı & 0,8 & 1,5007 \\
\hline ILMN_32388i & 23248 & RPRD2 & regulation of nuclear pre-mRNA domaiı & 0,8 & 0,0000 \\
\hline ILMN_18089: & 6194 & RPS6 & ribosomal protein S6 & 0,8 & 1,2499 \\
\hline ILMN_17383i & 1938 & EEF2 & eukaryotic translation elongation factol & 0,8 & 2,0799 \\
\hline ILMN_18007! & 6167 & RPL37 & ribosomal protein L37 & 0,8 & 2,6244 \\
\hline ILMN_23159" & 81606 & LBH & limb bud and heart development homo & 0,8 & 2,0799 \\
\hline ILMN_16523: & 222223 & KIAA1324L & KIAA1324-like & 0,8 & 2,6244 \\
\hline ILMN_17247: & 8440 & NCK2 & NCK adaptor protein 2 & 0,9 & 1,5007 \\
\hline ILMN_16593: & 26610 & ELP4 & elongation protein 4 homolog (S. cerev & 0,9 & 2,6244 \\
\hline ILMN_17375: & 6874 & TAF4 & TAF4 RNA polymerase II, TATA box binc & 0,9 & 1,8141 \\
\hline ILMN_17845! & 6383 & SDC2 & syndecan 2 & 0,9 & 2,6244 \\
\hline ILMN_17202، & 64806 & IL25 & interleukin 25 & 0,9 & 0,8244 \\
\hline ILMN_17189: & 9802 & DAZAP2 & DAZ associated protein 2 & 0,9 & 0,0000 \\
\hline ILMN_17490" & 3115 & HLA-DPB1 & major histocompatibility complex, class & 0,9 & 2,0799 \\
\hline ILMN_17629! & 9812 & KIAA0141 & KIAA0141 & 0,9 & 1,2499 \\
\hline ILMN_17719; & 57153 & SLC44A2 & solute carrier family 44 , member 2 & 0,9 & 1,8141 \\
\hline ILMN_17671، & 54816 & ZNF280D & zinc finger protein $280 D$ & 0,9 & 1,8141 \\
\hline ILMN_174211 & 83743 & GRWD1 & glutamate-rich WD repeat containing 1 & 0,9 & 1,0331 \\
\hline ILMN_16525: & 89853 & FAM125B & family with sequence similarity $125, \mathrm{~m} \epsilon$ & 0,9 & 1,2499 \\
\hline ILMN_17287i & 221395 & GPR116 & G protein-coupled receptor 116 & 0,9 & 2,6244 \\
\hline ILMN_17734" & 6764 & ST5 & suppression of tumorigenicity 5 & 0,9 & 2,0799 \\
\hline ILMN_16900! & 9270 & ITGB1BP1 & integrin beta 1 binding protein 1 & 0,9 & 2,6244 \\
\hline ILMN_17737: & 65005 & MRPL9 & mitochondrial ribosomal protein L9 & 0,9 & 2,6244 \\
\hline ILMN_21018: & 55353 & LAPTM4B & Iysosomal protein transmembrane 4 be & 0,9 & 1,8141 \\
\hline ILMN_17313! & 55486 & PARL & presenilin associated, rhomboid-like & 0,9 & 1,5007 \\
\hline ILMN_32395: & 401115 & C4orf48 & chromosome 4 open reading frame 48 & 0,9 & 2,6244 \\
\hline ILMN_17022: & 79630 & C1orf54 & chromosome 1 open reading frame 54 & 0,9 & 2,6244 \\
\hline ILMN_17791: & 23384 & CYTSA & sperm antigen with calponin homology & 0,9 & 2,0799 \\
\hline ILMN_17291: & 55069 & C7orf42 & transmembrane protein 248 & 0,9 & 2,6244 \\
\hline ILMN_16827: & 8463 & TEAD2 & TEA domain family member 2 & 0,9 & 1,0331 \\
\hline ILMN_17975| & 116841 & SNAP47 & synaptosomal-associated protein, $47 \mathrm{kD}$ & 0,9 & 2,0799 \\
\hline ILMN_16893i & 83443 & SF3B5 & splicing factor $3 b$, subunit $5,10 \mathrm{kDa}$ & 0,9 & 2,0799 \\
\hline
\end{tabular}




\begin{tabular}{|c|c|c|c|c|c|}
\hline ILMN_17397، & 126792 & B3GALT6 & UDP-Gal:betaGal beta 1,3-galactosyltra & 0,9 & 2,6244 \\
\hline ILMN_170631 & 51241 & C14orf112 & cox16 cytochrome c oxidase assembly & 0,9 & 2,6244 \\
\hline ILMN_176181 & 90411 & MCFD2 & multiple coagulation factor deficiency 2 & 0,9 & 2,0799 \\
\hline ILMN_171721 & 10544 & PROCR & protein $\mathrm{C}$ receptor, endothelial & 0,9 & 2,0799 \\
\hline ILMN_16830، & 5504 & PPP1R2 & protein phosphatase 1 , regulatory (inhi & 0,9 & 2,6244 \\
\hline ILMN_166631 & 93058 & COQ10A & coenzyme Q10 homolog A (S. cerevisiaє & 0,9 & 2,6244 \\
\hline ILMN_16796: & 27352 & SGSM3 & small G protein signaling modulator 3 & 0,9 & 1,8141 \\
\hline ILMN_17256، & 6612 & SUMO3 & SMT3 suppressor of mif two 3 homolog & 0,9 & 1,5007 \\
\hline ILMN_23521: & 2064 & ERBB2 & v-erb-b2 erythroblastic leukemia viral o & 0,9 & 1,5007 \\
\hline ILMN_16743i & 10971 & YWHAQ & tyrosine 3-monooxygenase/tryptophan & 0,9 & 1,5007 \\
\hline ILMN_21988: & 2230 & FDX1 & ferredoxin 1 & 0,9 & 2,0799 \\
\hline ILMN_16911: & 116092 & DNTTIP1 & deoxynucleotidyltransferase, terminal, & 0,9 & 0,8244 \\
\hline ILMN_24081I & 24144 & TFIP11 & tuftelin interacting protein 11 & 0,9 & 2,0799 \\
\hline ILMN_16765| & 5654 & HTRA1 & HtrA serine peptidase 1 & 0,9 & 0,8244 \\
\hline ILMN_16767! & 4236 & MFAP1 & microfibrillar-associated protein 1 & 0,9 & 2,0799 \\
\hline ILMN_16949: & 5780 & PTPN9 & protein tyrosine phosphatase, non-rec€ & 0,9 & 2,6244 \\
\hline ILMN_18019: & 10465 & PPIH & peptidylprolyl isomerase $\mathrm{H}$ (cyclophilin & 0,9 & 0,8244 \\
\hline ILMN_16784! & 1123 & CHN1 & chimerin (chimaerin) 1 & 0,9 & 2,6244 \\
\hline ILMN_18129| & 10695 & CNPY3 & canopy 3 homolog (zebrafish) & 0,9 & 2,0799 \\
\hline ILMN_17970" & 10436 & EMG1 & EMG1 nucleolar protein homolog (S. ce & 0,9 & 2,0799 \\
\hline ILMN_22343: & 80772 & GLTPD1 & glycolipid transfer protein domain cont & 0,9 & 1,0331 \\
\hline ILMN_20555: & 55790 & CSGALNACT1 & chondroitin sulfate $\mathrm{N}$-acetylgalactosam & 0,9 & 1,2499 \\
\hline ILMN_16702" & 26020 & LRP10 & low density lipoprotein receptor-relate & 0,9 & 0,8244 \\
\hline ILMN_16971I & 51759 & C9orf78 & chromosome 9 open reading frame 78 & 0,9 & 1,5007 \\
\hline ILMN_17032: & 112970 & KTI12 & KTI12 homolog, chromatin associated (! & 0,9 & 2,6244 \\
\hline ILMN_167721 & 11178 & LZTS1 & leucine zipper, putative tumor suppres! & 0,9 & 1,8141 \\
\hline ILMN_16934! & 23478 & SEC11A & SEC11 homolog A (S. cerevisiae) & 0,9 & 2,6244 \\
\hline ILMN_18075: & 55181 & C17orf71 & smg-8 homolog, nonsense mediated $\mathrm{m}$ & 0,9 & 1,8141 \\
\hline ILMN_17351! & 57019 & CIAPIN1 & cytokine induced apoptosis inhibitor 1 & 0,9 & 1,8141 \\
\hline ILMN_17200: & 30844 & EHD4 & EH-domain containing 4 & 0,9 & 1,2499 \\
\hline ILMN_17021" & 2739 & GLO1 & glyoxalase I & 0,9 & 2,6244 \\
\hline ILMN_16724، & 6135 & RPL11 & ribosomal protein L11 & 0,9 & 2,6244 \\
\hline ILMN_16878: & 22928 & SEPHS2 & selenophosphate synthetase 2 & 0,9 & 2,6244 \\
\hline ILMN_165151 & 8603 & FAM193A & family with sequence similarity $193, \mathrm{~m} \epsilon$ & 0,9 & 2,0799 \\
\hline ILMN_16692 & 7564 & ZNF16 & zinc finger protein 16 & 0,9 & 1,8141 \\
\hline ILMN_174331 & 7265 & TTC1 & tetratricopeptide repeat domain 1 & 0,9 & 1,0331 \\
\hline ILMN_16727! & 90550 & CCDC109A & mitochondrial calcium uniporter & 0,9 & 2,6244 \\
\hline ILMN_22255! & 27034 & ACAD8 & acyl-CoA dehydrogenase family, memb & 0,9 & 1,2499 \\
\hline ILMN_17163i & 64225 & ATL2 & atlastin GTPase 2 & 0,9 & 1,2499 \\
\hline ILMN_17553! & 7275 & TUB & tubby homolog (mouse) & 0,9 & 0,8244 \\
\hline ILMN_16573: & 5439 & POLR2J & polymerase (RNA) II (DNA directed) pol & 0,9 & 2,0799 \\
\hline ILMN_21148" & 6135 & RPL11 & ribosomal protein L11 & 0,9 & 2,6244 \\
\hline ILMN_32483، & 54617 & INO80 & INO80 homolog (S. cerevisiae) & 0,9 & 1,2499 \\
\hline ILMN_18067| & 140459 & ASB6 & ankyrin repeat and SOCS box containin & 0,9 & 2,6244 \\
\hline ILMN_16542i & 115 & ADCY9 & adenylate cyclase 9 & 0,9 & 1,2499 \\
\hline ILMN_17308: & 284424 & C19orf30 & MIR7-3 host gene (non-protein coding) & 0,9 & 2,0799 \\
\hline
\end{tabular}




\begin{tabular}{|c|c|c|c|c|c|}
\hline ILMN_17267| & 55748 & CNDP2 & CNDP dipeptidase 2 (metallopeptidase & 0,9 & 1,5007 \\
\hline ILMN_171281 & 126669 & SHE & Src homology 2 domain containing $\mathrm{E}$ & 0,9 & 2,0799 \\
\hline ILMN_17751| & 5136 & PDE1A & phosphodiesterase $1 \mathrm{~A}$, calmodulin-dep & 0,9 & 2,6244 \\
\hline ILMN_18123! & 9168 & TMSB10 & thymosin beta 10 & 0,9 & 1,8141 \\
\hline ILMN_17047: & 22918 & CD93 & CD93 molecule & 0,9 & 1,5007 \\
\hline ILMN_16759: & 3459 & IFNGR1 & interferon gamma receptor 1 & 0,9 & 1,8141 \\
\hline ILMN_16851| & 54942 & C9orf6 & family with sequence similarity $206, m \epsilon$ & 0,9 & 2,0799 \\
\hline ILMN_17041: & 207063 & DHRSX & dehydrogenase/reductase (SDR family) & 0,9 & 1,5007 \\
\hline ILMN_17901| & 3815 & KIT & v-kit Hardy-Zuckerman 4 feline sarcoma & 0,9 & 2,6244 \\
\hline ILMN_16886: & 10782 & ZNF274 & zinc finger protein 274 & 0,9 & 1,2499 \\
\hline ILMN_17601| & 6804 & STX1A & syntaxin $1 \mathrm{~A}$ (brain) & 0,9 & 1,8141 \\
\hline ILMN_16731: & 79042 & TSEN34 & tRNA splicing endonuclease 34 homolo: & 0,9 & 2,6244 \\
\hline ILMN_177381 & 7041 & TGFB1I1 & transforming growth factor beta 1 indu & 0,9 & 2,6244 \\
\hline ILMN_17244: & 23464 & GCAT & glycine C-acetyltransferase & 0,9 & 2,0799 \\
\hline ILMN_17076 & 10686 & CLDN16 & claudin 16 & 0,9 & 1,8141 \\
\hline
\end{tabular}




\section{Supplemental Figure 2}

\section{KEGG}

Up-regulated Transcripts Down-regulated Transcripts

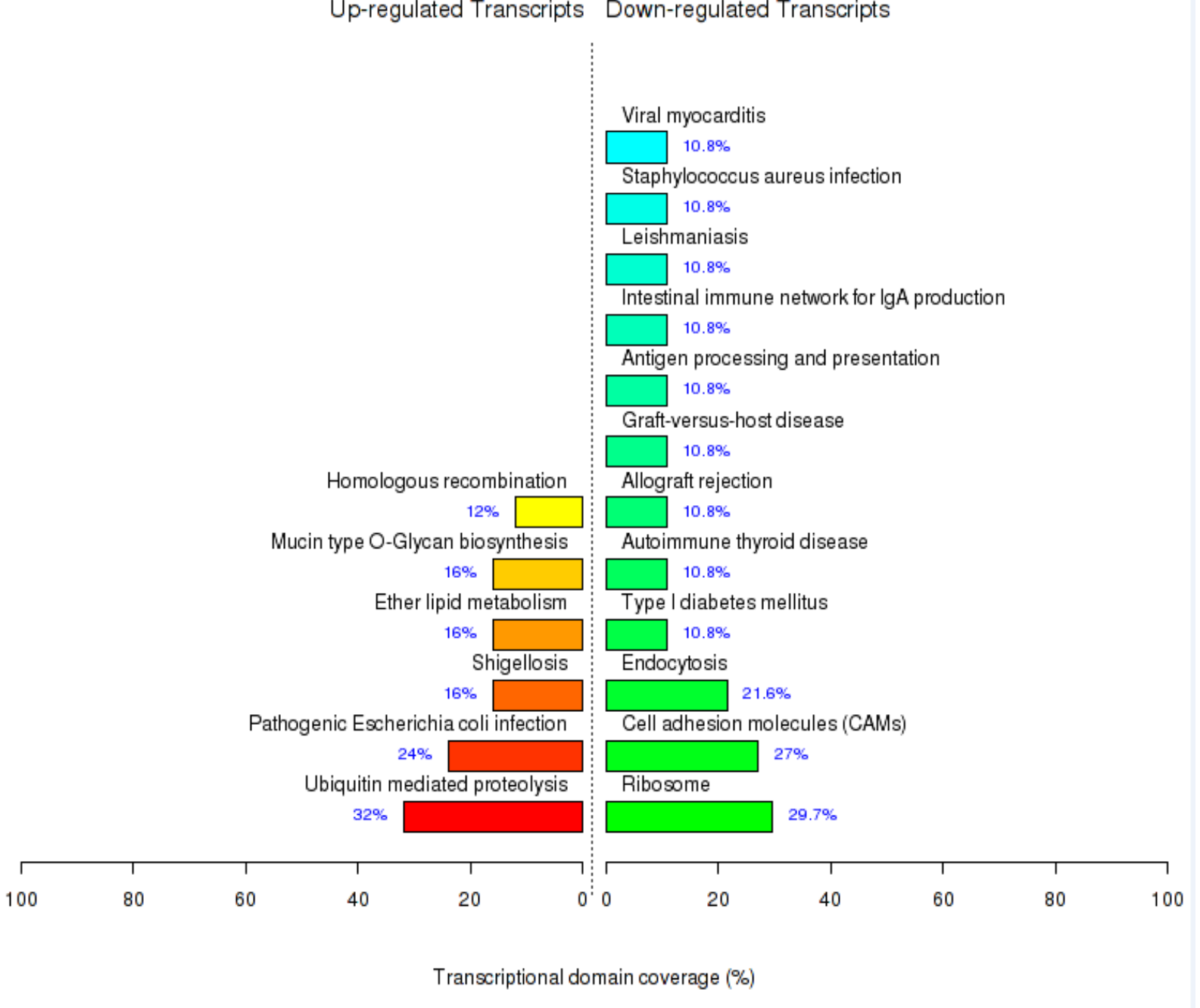




\section{Supplemental Figure 1}

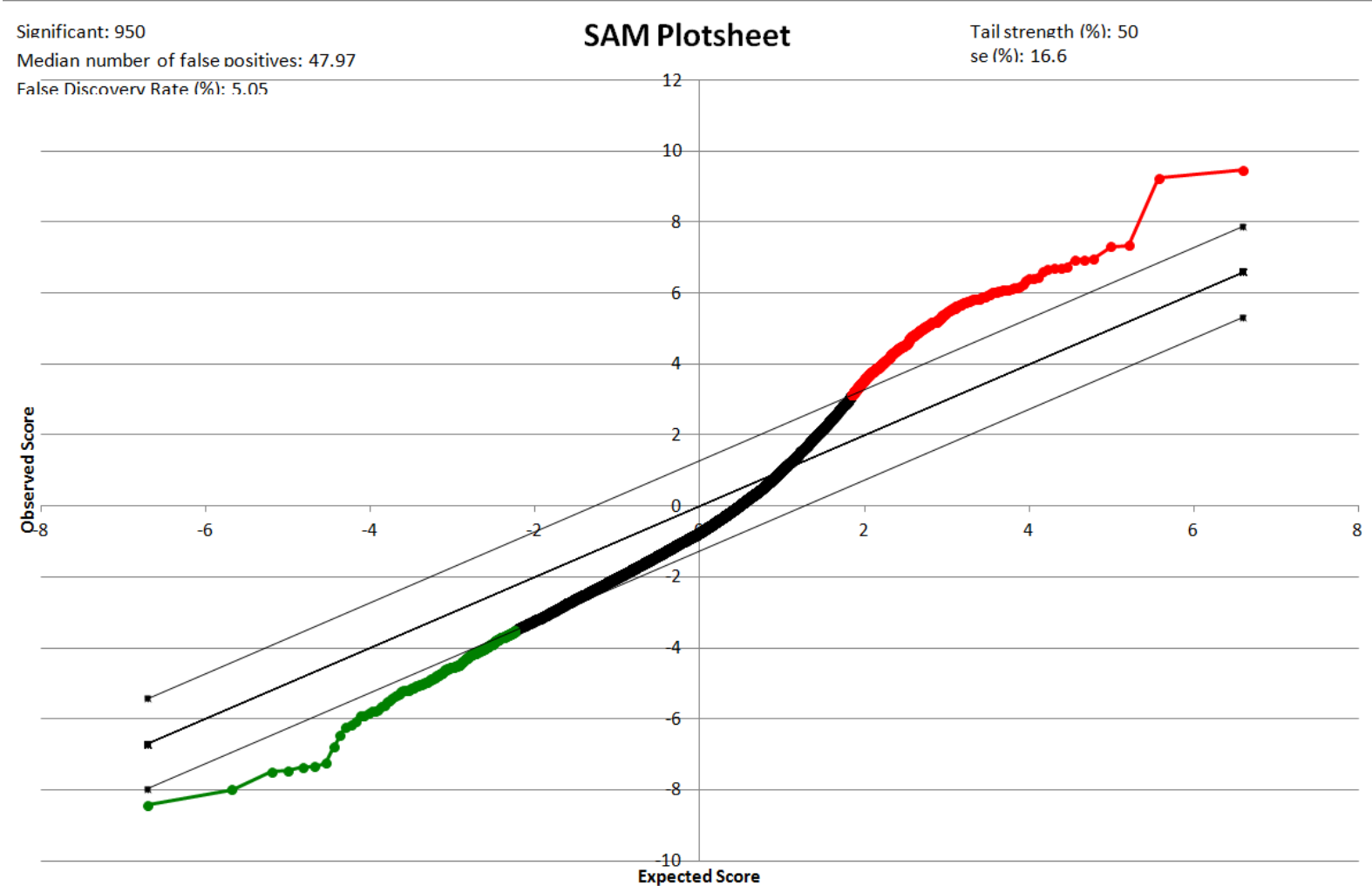

Expected Score

Down-regulated

Up-regulated 


\begin{tabular}{|c|c|c|c|c|}
\hline Gene ID ID & Gene ID & NAME & \multicolumn{2}{|c|}{ old Changy-value(\% } \\
\hline ILMN_20753: & 8364 HIST1H4C & histone cluster $1, \mathrm{H} 4 \mathrm{c}$ & 10,6 & 1,8141 \\
\hline ILMN_32514I & 64710 NUCKS1 & nuclear casein kinase and cyclin-depen & 8,6 & 1,0331 \\
\hline ILMN_21135: & 51449 PCYOX1 & prenylcysteine oxidase 1 & 7,7 & 1,5007 \\
\hline ILMN_21300 & 91368 CDKN2AIPNL & CDKN2A interacting protein $\mathrm{N}$-terminal & 7,7 & 0,3847 \\
\hline ILMN_16613: & 6711 SPTBN1 & spectrin, beta, non-erythrocytic 1 & 6,8 & 1,5007 \\
\hline ILMN_21159، & 84124 ZNF394 & zinc finger protein 394 & 6,3 & 1,8141 \\
\hline ILMN_24157، & 26118 WSB1 & WD repeat and SOCS box containing 1 & 6,3 & 1,2499 \\
\hline ILMN_20519| & 126272 EID2B & EP300 interacting inhibitor of different & 6,1 & 1,2499 \\
\hline ILMN_21137: & 157777 C8orf45 & minichromosome maintenance domair & 6,0 & 0,3847 \\
\hline ILMN_32371: & 84142 FAM175A & family with sequence similarity $175, \mathrm{~m} \iota$ & 6,0 & 0,6097 \\
\hline ILMN_22274! & 256051 ZNF549 & zinc finger protein 549 & 5,9 & 1,5007 \\
\hline ILMN_16921، & 7561 ZNF14 & zinc finger protein 14 & 5,8 & 0,3847 \\
\hline ILMN_23138i & 91120 ZNF682 & zinc finger protein 682 & 5,7 & 0,7969 \\
\hline ILMN_21768i & 7620 ZNF69 & zinc finger protein 69 & 5,7 & 1,8141 \\
\hline ILMN_21502! & 55033 FKBP14 & FK506 binding protein 14, 22 kDa & 5,7 & 1,5007 \\
\hline ILMN_22103i & 200728 TMEM17 & transmembrane protein 17 & 5,6 & 1,8141 \\
\hline ILMN_21107! & 1138 CHRNA5 & cholinergic receptor, nicotinic, alpha 5 & 5,6 & 1,5007 \\
\hline ILMN_17224! & 142679 DUSP19 & dual specificity phosphatase 19 & 5,6 & 1,8141 \\
\hline ILMN_22860: & 117155 CATSPER2 & eerm associated 2 & 5,5 & 0,3847 \\
\hline ILMN_22153i & 317781 DDX51 & DEAD (Asp-Glu-Ala-Asp) box polypeptic & 5,5 & 0,3847 \\
\hline ILMN_17350: & 1316 KLF6 & Kruppel-like $f$ & 5,5 & 1,5007 \\
\hline ILMN_32416: & 84109 QRFPR & pyroglutar & 5,5 & 0,7969 \\
\hline ILMN_21225: & 147372 CCBE1 & ding EGF dom: & 5,4 & 0,3847 \\
\hline ILMN_16615: & 54741 LEPROT & leptin receptor overlapping transcript & 5,4 & 0,6097 \\
\hline ILMN_21881: & 339231 ARL16 & ADP-ribosylation factor-like 16 & 5,3 & 0,3847 \\
\hline ILMN_17213، & 84061 MAGT1 & magnesiu & 5,3 & 1,0331 \\
\hline ILMN_32381i & 399761 BMS1P5 & BMS1 $p$ & 5,3 & 0,7969 \\
\hline ILMN_22061i & 57477 SHROOM4 & shroom family & 5,2 & 1,8141 \\
\hline ILMN_22357، & 56850 GRIPAP1 & GRIP1 associated protein 1 & 5,1 & 1,0331 \\
\hline ILMN_22049| & 7516 XRCC2 & X-ray repair complementing defective I & 5,0 & 1,5007 \\
\hline ILMN_23342، & 1385 CREB1 & CAMP responsive element binding prot & 5,0 & 1,2499 \\
\hline ILMN_22857: & 55775 TDP1 & tyrosyl-DNA phosphodiesterase 1 & 5,0 & 1,8141 \\
\hline ILMN_21543: & 9723 SEMA3E & sema domain, immunoglobulin domair & 4,9 & 2,0799 \\
\hline ILMN_22357i & 81033 KCNH6 & potassium voltage-gated channel, subf & 4,9 & 0,3847 \\
\hline ILMN_17510: & 162073 ITPRIPL2 & inositol 1,4,5-trisphosphate receptor in & 4,9 & 1,0331 \\
\hline ILMN_223011 & 641737 FLJ44124 & uncharacterized LOC641737 & 4,9 & 0,3847 \\
\hline ILMN_17857I & 56203 LMOD3 & leiomodin 3 (fetal) & 4,8 & 1,5007 \\
\hline ILMN_20787: & 84334 C14orf153 & apoptogenic 1 & 4,8 & 1,2499 \\
\hline ILMN_17104| & 5700 PSMC1 & proteasome (prosome, macropain) 26s & 4,8 & 0,7969 \\
\hline ILMN_20785، & 154791 HSPC268 & chromosome 7 open reading frame 55 & 4,7 & 1,0331 \\
\hline ILMN_216231 & 11144 DMC1 & DMC1 dosage suppressor of mck1 hom & 4,7 & 1,5007 \\
\hline ILMN_21948! & 649598 FLJ46309 & hypothetical protein LOC649598 & 4,7 & 0,3847 \\
\hline ILMN_21284: & 1601 DAB2 & disabled homolog 2, mitogen-responsii & 4,7 & 1,5007 \\
\hline ILMN_21233: & 64167 LRAP & endoplasmic reticulum aminopeptidass & 4,7 & 1,2499 \\
\hline ILMN_21175| & 64789 DEM1 & defects in morphology 1 homolog (S. ct & 4,7 & 1,2499 \\
\hline
\end{tabular}




\begin{tabular}{|c|c|c|c|c|}
\hline ILMN_20757! & 126205 NLRP8 & NLR family, pyrin domain containing 8 & 4,7 & 0,3847 \\
\hline ILMN_17724! & 92014 MCART1 & solute carrier family 25, member 51 & 4,6 & 0,3847 \\
\hline ILMN_173601 & 79693 YRDC & yrdC domain containing (E. coli) & 4,5 & 0,7969 \\
\hline ILMN_16914: & 5718 PSMD12 & proteasome (prosome, macropain) $26 \varsigma$ & 4,5 & 0,6097 \\
\hline ILMN_16797: & 51449 PCYOX1 & prenylcysteine oxidase 1 & 4,4 & 1,5007 \\
\hline ILMN_20911: & 80867 HCG2P7 & HLA complex group 2 pseudogene 7 & 4,3 & 1,2499 \\
\hline ILMN_21397| & 22998 LIMCH1 & LIM and calponin homology domains 1 & 4,3 & 1,5007 \\
\hline ILMN_204931 & 151194 FAM119A & methyltransferase like $21 \mathrm{~A}$ & 4,2 & 1,2499 \\
\hline ILMN_236111 & 64393 ZMAT3 & zinc finger, matrin-type 3 & 4,2 & 1,5007 \\
\hline ILMN_173741 & 1316 KLF6 & Kruppel-like factor 6 & 4,2 & 2,0799 \\
\hline ILMN_16802 & 25862 USP49 & ubiquitin specific peptidase 49 & 4,1 & 0,3847 \\
\hline ILMN_32354: & 84278 HIATL2 & hippocampus abundant transcript-like & 4,1 & 1,0331 \\
\hline ILMN_20735، & 25764 C15orf63 & chromosome 15 open reading frame 6: & 4,1 & 1,2499 \\
\hline ILMN_211781 & 503632 DUXAP3 & double homeobox A pseudogene 3 & 4,1 & 1,0331 \\
\hline ILMN_21504! & 64417 C5orf28 & chromosome 5 open reading frame 28 & 4,1 & 1,2499 \\
\hline ILMN_22798: & 158399 ZNF483 & zinc finger protein 483 & 4,1 & 1,5007 \\
\hline ILMN_24078! & 54756 IL17RD & interleukin 17 receptor D & 4,0 & 0,7969 \\
\hline ILMN_21066! & 8548 BLZF1 & basic leucine zipper nuclear factor 1 & 4,0 & 1,5007 \\
\hline ILMN_324561 & 147172 LRRC37B2 & leucine rich repeat containing 37B pseı & 4,0 & 1,0331 \\
\hline ILMN_168011 & 10087 COL4A3BP & collagen, type IV, alpha 3 (Goodpasture & 4,0 & 1,2499 \\
\hline ILMN_322511 & 148203 ZNF738 & zinc finger protein 738 & 4,0 & 1,0331 \\
\hline ILMN_227931 & 2204 FCAR & Fc fragment of IgA, receptor for & 4,0 & 2,6244 \\
\hline ILMN_22494" & 10558 SPTLC1 & serine palmitoyltransferase, long chain & 4,0 & 0,3847 \\
\hline ILMN_16937i & 250 ALPP & hatase, placental & 3,9 & 1,2499 \\
\hline ILMN_16817| & 2309 FOXO3 & forkhead box $\mathrm{O} 3$ & 3,9 & 1,8141 \\
\hline ILMN_32443: & 202243 CCDC125 & coiled-coil domain containing 125 & 3,9 & 0,6097 \\
\hline ILMN_24011! & 9698 PUM1 & o homolog 1 (Drosophila) & 3,9 & 1,2499 \\
\hline ILMN_22767! & 23509 POFUT1 & protein O-fucosyltransferase 1 & 3,9 & 1,8141 \\
\hline ILMN_23701: & 3192 HNRNPU & heterogeneous nuclear ribonucleoprot & 3,8 & 1,2499 \\
\hline ILMN_17449، & 22836 RНОВТВ3 & Rho-related BTB domain containing 3 & 3,8 & 1,5007 \\
\hline ILMN_181211 & 8396 PIP5K2B & phosphatidylinositol-5-phosphate 4-kir & 3,7 & 0,3847 \\
\hline ILMN_21066! & 8548 BLZF1 & basic leucine zipper nuclear factor 1 & 3,7 & 0,7969 \\
\hline ILMN_32363" & 205327 C2orf69 & chromosome 2 open reading frame 69 & 3,7 & 0,7969 \\
\hline ILMN_23815: & 27297 CRCP & CGRP receptor component & 3,7 & 0,3847 \\
\hline ILMN_22117: & 400866 C21orf24 & long intergenic non-protein coding RN/ & 3,6 & 1,2499 \\
\hline ILMN_20591: & 79939 SLC35E1 & solute carrier family 35, member E1 & 3,6 & 0,3847 \\
\hline ILMN_18141! & 6925 TCF4 & transcription factor 4 & 3,6 & 1,2499 \\
\hline ILMN_16716| & 51478 HSD17B7 & hydroxysteroid (17-beta) dehydrogena & 3,6 & 1,2499 \\
\hline ILMN_17985i & 84515 MCM8 & minichromosome maintenance comple & 3,6 & 0,3847 \\
\hline ILMN_16977! & 55333 SYNJ2BP & synaptojanin 2 binding protein & 3,6 & 1,2499 \\
\hline ILMN_20545! & 285605 DTWD2 & DTW domain containing 2 & 3,6 & 0,3847 \\
\hline ILMN_33078 & 54058 C21orf58 & chromosome 21 open reading frame $5 \vdots$ & 3,5 & 0,6097 \\
\hline ILMN_16894! & 26137 ZBTB20 & zinc finger and BTB domain containing & 3,5 & 1,0331 \\
\hline ILMN_21235! & 374986 FAM73A & family with sequence similarity 73, meı & 3,5 & 0,3847 \\
\hline ILMN_23342، & 1385 CREB1 & CAMP responsive element binding prot & 3,5 & 0,6097 \\
\hline ILMN_20549: & 63929 XPNPEP3 & X-prolyl aminopeptidase (aminopeptidi & 3,4 & 1,5007 \\
\hline
\end{tabular}




\begin{tabular}{|c|c|c|c|c|}
\hline ILMN_23304! & 54940 OCIAD1 & OCIA domain containing 1 & 3,4 & 0,3847 \\
\hline ILMN_22128: & 84765 ZNF577 & zinc finger protein 577 & 3,4 & 1,5007 \\
\hline ILMN_20790! & 58493 C9orf80 & chromosome 9 open reading frame 80 & 3,4 & 0,6097 \\
\hline ILMN_23424! & 27068 PPA2 & pyrophosphatase (inorganic) 2 & 3,3 & 0,3847 \\
\hline ILMN_20910: & 157657 C8orf37 & chromosome 8 open reading frame 37 & 3,3 & 0,3847 \\
\hline ILMN_22884i & 84984 C3orf34 & centrosomal protein $19 \mathrm{kDa}$ & 3,3 & 1,5007 \\
\hline ILMN_22622: & 10206 TRIM13 & tripartite motif containing 13 & 3,3 & 1,5007 \\
\hline ILMN_21581| & 80264 ZNF430 & zinc finger protein 430 & 3,3 & 0,7969 \\
\hline ILMN_16980: & 10395 DLC1 & deleted in liver cancer 1 & 3,3 & 1,0331 \\
\hline ILMN_16719| & 7360 UGP2 & UDP-glucose pyrophosphorylase 2 & 3,2 & 0,7969 \\
\hline ILMN_21553: & 22834 ZNF652 & zinc finger protein 652 & 3,2 & 0,3847 \\
\hline ILMN_17267i & 246243 RNASEH1 & ribonuclease $\mathrm{H} 1$ & 3,2 & 2,0799 \\
\hline ILMN_32517: & 87178 PNPT1 & polyribonucleotide nucleotidyltransferi & 3,2 & 0,7969 \\
\hline ILMN_16559: & 9701 SAPS2 & protein phosphatase 6 , regulatory subı & 3,1 & 1,8141 \\
\hline ILMN_207331 & 3586 IL10 & interleukin 10 & 3,1 & 0,3847 \\
\hline ILMN_32492، & 113277 TMEM106A & transmembrane protein $106 \mathrm{~A}$ & 3,1 & 0,3847 \\
\hline ILMN_18126: & $4641 \mathrm{MYO1C}$ & myosin IC & 3,1 & 0,3847 \\
\hline ILMN_21809: & 387700 SLC16A12 & solute carrier family 16, member 12 (m & 3,1 & 0,3847 \\
\hline ILMN_22435! & 10838 ZNF275 & zinc finger protein 275 & 3,0 & 0,3847 \\
\hline ILMN_16615i & 977 CD151 & CD151 molecule (Raph blood group) & 3,0 & 0,7969 \\
\hline ILMN_21052! & 145482 PTGR2 & prostaglandin reductase 2 & 3,0 & 0,3847 \\
\hline ILMN_21689! & 8562 DENR & density-regulated protein & 3,0 & 0,6097 \\
\hline ILMN_177821 & 285966 FLJ40722 & family with sequence similarity $115, \mathrm{~m} \iota$ & 3,0 & 0,3847 \\
\hline ILMN_17863، & 3842 TNPO1 & transportin 1 & 3,0 & 2,6244 \\
\hline ILMN_20535: & 54933 RHBDL2 & rhomboid, veinlet-like 2 (Drosophila) & 2,9 & 0,3847 \\
\hline ILMN_18056I & 90649 ZNF486 & zinc finger protein 486 & 2,9 & 0,3847 \\
\hline ILMN_222211 & 55728 N4BP2 & D4 binding protein 2 & 2,9 & 0,3847 \\
\hline ILMN_20553: & 8930 MBD4 & methyl-CpG binding domain protein 4 & 2,9 & 1,2499 \\
\hline ILMN_32450! & 50807 ASAP1 & ArfGAP with $\mathrm{SH} 3$ domain, ankyrin repe & 2,8 & 1,8141 \\
\hline ILMN_17137| & 136051 ZNF786 & zinc finger protein 786 & 2,8 & 0,3847 \\
\hline ILMN_23974i & 10052 GJC1 & gap junction protein, gamma $1,45 \mathrm{kDa}$ & 2,8 & 0,7969 \\
\hline ILMN_16943! & 3382 ICA1 & islet cell autoantigen $1,69 \mathrm{kDa}$ & 2,8 & 0,3847 \\
\hline ILMN_17636! & 89970 RSPRY1 & ring finger and SPRY domain containin६ & 2,8 & 1,8141 \\
\hline ILMN_322661 & 642280 MGC26356 & zinc finger protein 876, pseudogene & 2,8 & 0,7969 \\
\hline ILMN_16810: & 10555 AGPAT2 & 1-acylglycerol-3-phosphate O-acyltrans & 2,7 & 1,8141 \\
\hline ILMN_16919، & $10983 \mathrm{CCNI}$ & cyclin I & 2,7 & 1,0331 \\
\hline ILMN_17612 & 5143 PDE4C & phosphodiesterase 4C, cAMP-specific & 2,7 & 0,3847 \\
\hline ILMN_172121 & 1438 CSF2RA & colony stimulating factor 2 receptor, al & 2,7 & 0,3847 \\
\hline ILMN_23633! & 8740 TNFSF14 & tumor necrosis factor (ligand) superfan & 2,7 & 1,0331 \\
\hline ILMN_238311 & 27072 VPS41 & vacuolar protein sorting 41 homolog (S & 2,7 & 0,3847 \\
\hline ILMN_21050: & 26258 PLDN & pallidin homolog (mouse) & 2,6 & 0,3847 \\
\hline ILMN_16670: & 55066 PDPR & pyruvate dehydrogenase phosphatase & 2,6 & 1,8141 \\
\hline ILMN_20891: & 94056 SYAP1 & synapse associated protein 1 & 2,6 & 0,3847 \\
\hline ILMN_16901: & 401494 PTPLAD2 & protein tyrosine phosphatase-like $A$ do & 2,6 & 0,3847 \\
\hline ILMN_16590! & 9475 ROCK2 & Rho-associated, coiled-coil containing & 2,6 & 1,5007 \\
\hline ILMN_24056i & 330 BIRC3 & baculoviral IAP repeat containing 3 & 2,6 & 1,5007 \\
\hline
\end{tabular}




\begin{tabular}{|c|c|c|c|c|}
\hline ILMN_325161 & 54813 KLHL28 & kelch-like 28 (Drosophila) & 2,6 & 1,0331 \\
\hline ILMN_17110 & 653489 LOC653489 & RANBP2-like and GRIP domain containi & 2,5 & 0,7969 \\
\hline ILMN_180851 & 30011 SH3KBP1 & SH3-domain kinase binding protein 1 & 2,5 & 1,8141 \\
\hline ILMN_205141 & 87178 PNPT1 & polyribonucleotide nucleotidyltransferi & 2,5 & 0,6097 \\
\hline ILMN_21750! & 56165 TDRD1 & tudor domain containing 1 & 2,5 & 2,0799 \\
\hline ILMN_181421 & 54943 C21orf55 & DnaJ (Hsp40) homolog, subfamily C, mı & 2,5 & 0,7969 \\
\hline ILMN_22435: & 79864 C11orf63 & chromosome 11 open reading frame 6: & 2,5 & 0,3847 \\
\hline ILMN_23169" & 10859 LILRB1 & leukocyte immunoglobulin-like receptc & 2,4 & 1,0331 \\
\hline ILMN_23764! & 1438 CSF2RA & colony stimulating factor 2 receptor, al & 2,4 & 0,6097 \\
\hline ILMN_32402: & 140771 SMCR5 & Smith-Magenis syndrome chromosome & 2,4 & 0,3847 \\
\hline ILMN_17306 & 374860 ANKRD30B & ankyrin repeat domain $30 \mathrm{~B}$ & 2,4 & 1,0331 \\
\hline ILMN_17784! & 3606 IL18 & interleukin 18 (interferon-gamma-indu & 2,4 & 1,0331 \\
\hline ILMN_22203: & 285855 RPL7L1 & ribosomal protein L7-like 1 & 2,4 & 0,7969 \\
\hline ILMN_17367| & 226 ALDOA & aldolase A, fructose-bisphosphate & 2,4 & 2,6244 \\
\hline ILMN_21610| & 126626 GABPB2 & GA binding protein transcription factor & 2,4 & 0,7969 \\
\hline ILMN_24019| & 146059 CDAN1 & codanin 1 & 2,4 & 0,6097 \\
\hline ILMN_22281! & 221322 C6orf170 & chromosome 6 open reading frame 171 & 2,4 & 0,7969 \\
\hline ILMN_181311 & 117248 GALNTL2 & UDP-N-acetyl-alpha-D-galactosamine:p & 2,4 & 2,6244 \\
\hline ILMN_18155" & 7766 ZNF223 & zinc finger protein 223 & 2,3 & 0,3847 \\
\hline ILMN_21016! & 54799 MBTD1 & mbt domain containing 1 & 2,3 & 2,6244 \\
\hline ILMN_16914: & 4826 NNAT & neurona & 2,3 & 2,0799 \\
\hline ILMN_21612i & 57464 FAM40B & family with sequence similarity 40, meı & 2,3 & 0,3847 \\
\hline ILMN_21063i & 9966 TNFSF15 & tumor necrosis factor (ligand) superfan & 2,3 & 1,5007 \\
\hline ILMN_21522! & 6752 SSTR2 & somatostatin receptor 2 & 2,3 & 1,2499 \\
\hline ILMN_22732: & 57835 SLC4A5 & solute carrier family 4 , sodium bicarbol & 2,3 & 0,3847 \\
\hline ILMN_24047! & 6817 SULT1A1 & sulfotransferase family, cytosolic, $1 \mathrm{~A}, \mathrm{p}$ & 2,3 & 0,0000 \\
\hline ILMN_18039: & 286451 YIPF6 & Yip1 domain family, member 6 & 2,3 & 1,2499 \\
\hline ILMN_32601i & 401082 FLJ25363 & uncharacterized LOC401082 & 2,3 & 0,7969 \\
\hline ILMN_20937، & 79862 ZNF669 & zinc finger protein 669 & 2,3 & 0,3847 \\
\hline ILMN_16534: & 84839 RAXL1 & retina and anterior neural fold homeok & 2,2 & 0,3847 \\
\hline ILMN_17451: & 23543 RBM9 & RNA binding protein, fox-1 homolog (C & 2,2 & 1,5007 \\
\hline ILMN_17601: & 64121 RRAGC & Ras-related GTP binding C & 2,2 & 1,2499 \\
\hline ILMN_22249| & 201895 C4orf34 & chromosome 4 open reading frame 34 & 2,2 & 1,8141 \\
\hline ILMN_22099! & 440503 PLIN5 & perilipin 5 & 2,2 & 0,3847 \\
\hline ILMN_17384i & 55142 CEP27 & HAUS augmin-like complex, subunit 2 & 2,2 & 0,7969 \\
\hline ILMN_20974: & 27071 DAPP1 & dual adaptor of phosphotyrosine and 3 & 2,2 & 0,6097 \\
\hline ILMN_210621 & 284161 GDPD1 & glycerophosphodiester phosphodiester & 2,2 & 1,5007 \\
\hline ILMN_17811! & 4067 LYN & v-yes-1 Yamaguchi sarcoma viral relate & 2,2 & 1,8141 \\
\hline ILMN_22330! & 26279 PLA2G2D & phospholipase A2, group IID & 2,2 & 0,3847 \\
\hline ILMN_22615: & 326 AIRE & autoimmune regulator & 2,2 & 0,7969 \\
\hline ILMN_20933i & 6617 SNAPC1 & small nuclear RNA activating complex, | & 2,2 & 1,0331 \\
\hline ILMN_16884! & 8073 PTP4A2 & protein tyrosine phosphatase type IVA, & 2,2 & 0,3847 \\
\hline ILMN_20488: & 80224 NUBPL & nucleotide binding protein-like & 2,1 & 0,3847 \\
\hline ILMN_16766| & 404093 CUEDC1 & CUE domain containing 1 & 2,1 & 0,7969 \\
\hline ILMN_17673! & 367 AR & androgen receptor & 2,1 & 1,5007 \\
\hline ILMN_18112: & 160728 SLC5A8 & solute carrier family 5 (iodide transpor & 2,1 & 0,3847 \\
\hline
\end{tabular}




\begin{tabular}{|c|c|c|c|c|}
\hline ILMN_22841i & 7360 UGP2 & UDP-glucose pyrophosphorylase 2 & 2,1 & 2,6244 \\
\hline ILMN_16562، & 79827 ASAM & CXADR-like membrane protein & 2,1 & 2,0799 \\
\hline ILMN_18140 & 5256 PHKA2 & phosphorylase kinase, alpha 2 (liver) & 2,1 & 0,6097 \\
\hline ILMN_178721 & 84858 ZNF503 & zinc finger protein 503 & 2,1 & 2,6244 \\
\hline ILMN_20478i & 56127 PCDHB9 & protocadherin beta 9 & 2,1 & 0,3847 \\
\hline ILMN_22465، & 653399 GSTTP2 & glutathione S-transferase theta pseudc & 2,1 & 0,7969 \\
\hline ILMN_20700، & 152926 PPM1K & protein phosphatase, $\mathrm{Mg} 2+/ \mathrm{Mn} 2+$ dep & 2,1 & 1,2499 \\
\hline ILMN_23573i & 2218 FKTN & fukutin & 2,1 & 0,7969 \\
\hline ILMN_18010، & 6693 SPN & sialophorin & 2,0 & 2,0799 \\
\hline ILMN_32393، & 442578 STAG3L3 & stromal antigen 3-like 3 & 2,0 & 1,5007 \\
\hline ILMN_20969" & 140469 МYO3В & myosin IIIB & 2,0 & 1,5007 \\
\hline ILMN_167601 & 22982 DIP2C & DIP2 disco-interacting protein 2 homol & 2,0 & 2,6244 \\
\hline ILMN_18126، & 196 AHR & aryl hydrocarbon receptor & 2,0 & 0,7969 \\
\hline ILMN_16957: & 27161 EIF2C2 & eukaryotic translation initiation factor : & 2,0 & 2,0799 \\
\hline ILMN_324641 & 3187 HNRNPH1 & heterogeneous nuclear ribonucleoprot & 2,0 & 0,7969 \\
\hline ILMN_229701 & 9747 FAM115A & family with sequence similarity $115, \mathrm{~m} \iota$ & 2,0 & 1,5007 \\
\hline ILMN_17561! & 79157 MFSD11 & major facilitator superfamily domain cc & 2,0 & 1,2499 \\
\hline ILMN_17267i & 23112 TNRC6B & trinucleotide repeat containing 6B & 2,0 & 0,3847 \\
\hline ILMN_22609! & 706 TSPO & translocator protein (18kDa) & 2,0 & 1,8141 \\
\hline ILMN_17551: & 440275 EIF2AK4 & eukaryotic translation initiation factor: & 2,0 & 0,3847 \\
\hline ILMN_17463: & 7813 EVI5 & ecotropic viral integration site 5 & 2,0 & 1,0331 \\
\hline ILMN_18061: & $57680 \mathrm{CHD} 8$ & chromodomain helicase DNA binding $p$ & 2,0 & 1,2499 \\
\hline ILMN_17975: & 1845 DUSP3 & dual specificity phosphatase 3 & 2,0 & 1,8141 \\
\hline ILMN_23219: & 64421 DCLRE1C & DNA cross-link repair $1 \mathrm{C}$ & 2,0 & 0,3847 \\
\hline ILMN_24061: & 11025 LILRB3 & leukocyte immunoglobulin-like receptc & 2,0 & 0,7969 \\
\hline ILMN_22747 & 80736 SLC44A4 & solute carrier family 44, member 4 & 2,0 & 1,2499 \\
\hline ILMN_232401 & 800 CALD1 & caldesmon 1 & 1,9 & 1,8141 \\
\hline ILMN_16893: & 9891 NUAK1 & NUAK family, SNF1-like kinase, 1 & 1,9 & 1,2499 \\
\hline ILMN_17913: & 442582 STAG3L2 & stromal antigen 3-like 2 & 1,9 & 1,2499 \\
\hline ILMN_17080। & 4134 MAP4 & microtubule-associated protein 4 & 1,9 & 0,3847 \\
\hline ILMN_23522، & 166824 RASSF6 & Ras association (RalGDS/AF-6) domain & 1,9 & 0,7969 \\
\hline ILMN_17770، & 90338 ZNF160 & zinc finger protein 160 & 1,9 & 0,3847 \\
\hline ILMN_32392: & 6085 RNY3 & RNA, Ro-associated Y3 & 1,9 & 0,6097 \\
\hline ILMN_21482! & 10081 PDCD7 & programmed cell death 7 & 1,9 & 1,2499 \\
\hline ILMN_18150: & 5292 PIM1 & pim-1 oncogene & 1,9 & 1,8141 \\
\hline ILMN_17956! & 8073 РTP4A2 & protein tyrosine phosphatase type IVA, & 1,9 & 1,8141 \\
\hline ILMN_17146: & 27327 TNRC6A & trinucleotide repeat containing $6 \mathrm{~A}$ & 1,9 & 1,2499 \\
\hline ILMN_21824i & 79801 SHCBP1 & SHC SH2-domain binding protein 1 & 1,9 & 1,0331 \\
\hline ILMN_18156! & 10955 SERINC3 & serine incorporator 3 & 1,9 & 1,2499 \\
\hline ILMN_32285: & 84222 TMEM191A & transmembrane protein 191A (pseudo) & 1,9 & 0,3847 \\
\hline ILMN_32696! & 255031 FLJ35390 & uncharacterized LOC255031 & 1,9 & 0,3847 \\
\hline ILMN_178911 & 3652 IPP & intracisternal A particle-promoted poly & 1,9 & 0,7969 \\
\hline ILMN_17217: & 5469 PPARBP & mediator complex subunit 1 & 1,9 & 2,0799 \\
\hline ILMN_17590। & 115509 ZNF689 & zinc finger protein 689 & 1,9 & 2,6244 \\
\hline ILMN_172201 & 55156 ARMC1 & armadillo repeat containing 1 & 1,9 & 2,0799 \\
\hline ILMN_20646! & 91966 CXorf40A & chromosome $X$ open reading frame 40 & 1,9 & 2,0799 \\
\hline
\end{tabular}




\begin{tabular}{|c|c|c|c|c|}
\hline ILMN_17536: & 3202 HOXA5 & homeobox A5 & 1,9 & 0,3847 \\
\hline ILMN_16904، & 145438 C14orf82 & FRMD6 antisense RNA 1 (non-protein c & 1,8 & 0,3847 \\
\hline ILMN_17523: & 79939 SLC35E1 & solute carrier family 35, member E1 & 1,8 & 2,0799 \\
\hline ILMN_16913| & 6772 STAT1 & signal transducer and activator of trans & 1,8 & 2,0799 \\
\hline ILMN_23962 & 27250 PDCD4 & programmed cell death 4 (neoplastic tr & 1,8 & 0,7969 \\
\hline ILMN_23360! & 55714 ODZ3 & odz, odd Oz/ten-m homolog 3 (Drosop & 1,8 & 2,6244 \\
\hline ILMN_17489| & 83734 ATG10 & autophagy related 10 & 1,8 & 1,8141 \\
\hline ILMN_22259" & 2730 GCLM & glutamate-cysteine ligase, modifier suk & 1,8 & 1,2499 \\
\hline ILMN_17656! & 10056 FARSLB & phenylalanyl-tRNA synthetase, beta sul & 1,8 & 1,0331 \\
\hline ILMN_235141 & 50863 NTM & neurotrimin & 1,8 & 1,8141 \\
\hline ILMN_17386 & 286354 C9orf130 & chromosome 9 open reading frame 131 & 1,8 & 0,7969 \\
\hline ILMN_23461: & 79230 ZNF557 & zinc finger protein 557 & 1,8 & 0,3847 \\
\hline ILMN_32266، & 11145 PLA2G16 & phospholipase A2, group XVI & 1,8 & 2,0799 \\
\hline ILMN_20926! & 124801 LSM12 & LSM12 homolog (S. cerevisiae) & 1,8 & 1,8141 \\
\hline ILMN_17031: & 57226 LYRM2 & LYR motif containing 2 & 1,8 & 0,3847 \\
\hline ILMN_17873: & 65068 ALS2CR14 & amyotrophic lateral sclerosis 2 (juvenilı & 1,8 & 0,3847 \\
\hline ILMN_18094: & 10357 HMGB1L1 & high mobility group box 1 pseudogene & 1,7 & 2,6244 \\
\hline ILMN_18039! & 51768 TM7SF3 & transmembrane 7 superfamily membe & 1,7 & 1,5007 \\
\hline ILMN_22708، & 54663 WDR74 & WD repeat domain 74 & 1,7 & 1,0331 \\
\hline ILMN_17026: & 84617 TUBB6 & tubulin, beta 6 class $V$ & 1,7 & 0,7969 \\
\hline ILMN_21572: & 6718 AKR1D1 & aldo-keto reductase family 1 , member & 1,7 & 1,8141 \\
\hline ILMN_17978: & 23512 SUZ12 & suppressor of zeste 12 homolog (Droso & 1,7 & 1,2499 \\
\hline ILMN_16790: & 81931 ZNF93 & zinc finger protein 93 & 1,7 & 0,3847 \\
\hline ILMN_21754 & 54516 MTRF1L & mitochondrial translational release fac & 1,7 & 0,7969 \\
\hline ILMN_23626i & 8824 CES2 & carboxylesterase 2 & 1,7 & 0,6097 \\
\hline ILMN_17091| & 497262 RUNDC2C & RUN domain containi & 1,7 & 0,3847 \\
\hline ILMN_23941: & 55471 PRO1853 & chromosome 2 open reading frame 56 & 1,7 & 0,3847 \\
\hline ILMN_16798: & 51186 WBP5 & WW domain binding protein 5 & 1,7 & 0,7969 \\
\hline ILMN_22194: & 79056 PRRG4 & proline rich Gla (G-carboxyglutamic aci & 1,7 & 0,7969 \\
\hline ILMN_23705 & 54739 XAF1 & XIAP associated factor 1 & 1,7 & 1,5007 \\
\hline ILMN_33067: & 54502 RBM47 & RNA binding motif protein 47 & 1,7 & 1,2499 \\
\hline ILMN_18155" & 3203 HOXA6 & homeobox A6 & 1,7 & 0,7969 \\
\hline ILMN_23937| & 10093 ARPC4 & actin related protein $2 / 3$ complex, subı & 1,7 & 2,0799 \\
\hline ILMN_16575، & 91057 CCDC34 & coiled-coil domain containing 34 & 1,7 & 1,0331 \\
\hline ILMN_24144: & 5935 RBM3 & RNA binding motif (RNP1, RRM) proteiı & 1,7 & 0,3847 \\
\hline ILMN_21802: & 9980 DOPEY2 & dopey family member 2 & 1,7 & 1,8141 \\
\hline ILMN_218821 & 9140 ATG12 & autophagy related 12 & 1,6 & 0,7969 \\
\hline ILMN_16615: & 9729 KIAA0408 & KIAA0408 & 1,6 & 1,0331 \\
\hline ILMN_23876! & 1235 CCR6 & chemokine ( $\mathrm{C}-\mathrm{C}$ motif) receptor 6 & 1,6 & 2,0799 \\
\hline ILMN_33014| & 729324 LOC729324 & hCG1986447 & 1,6 & 1,2499 \\
\hline ILMN_24080: & 1936 EEF1D & eukaryotic translation elongation facto & 1,6 & 2,6244 \\
\hline ILMN_170921 & 81790 RNF170 & ring finger protein 170 & 1,6 & 1,5007 \\
\hline ILMN_20583: & 9475 ROCK2 & Rho-associated, coiled-coil containing $\mathrm{k}$ & 1,6 & 1,8141 \\
\hline ILMN_168531 & 374928 ZNF773 & zinc finger protein 773 & 1,6 & 0,3847 \\
\hline ILMN_22150| & 23594 ORC6L & origin recognition complex, subunit 6 & 1,6 & 1,0331 \\
\hline ILMN_20917I & 123688 LOC123688 & aminoglycoside phosphotransferase dc & 1,6 & 1,8141 \\
\hline
\end{tabular}




\begin{tabular}{|c|c|c|c|c|}
\hline ILMN_17830| & 788 SLC25A20 & solute carrier family 25 (carnitine/acylc & 1,6 & 0,3847 \\
\hline ILMN_32453i & 344787 ZNF860 & zinc finger protein 860 & 1,6 & 0,6097 \\
\hline ILMN_17606، & 3200 HOXA3 & homeobox A3 & 1,6 & 2,0799 \\
\hline ILMN_21337i & 399967 PATE2 & prostate and testis expressed 2 & 1,6 & 0,7969 \\
\hline ILMN_23648: & 8473 OGT & O-linked N-acetylglucosamine (GlcNAc) & 1,6 & 1,0331 \\
\hline ILMN_176951 & 54965 PIGX & phosphatidylinositol glycan anchor bio: & 1,6 & 0,3847 \\
\hline ILMN_16853" & 25870 SUMF2 & sulfatase modifying factor 2 & 1,6 & 2,0799 \\
\hline ILMN_23790: & 84901 NFATC2IP & nuclear factor of activated T-cells, cyto & 1,6 & 0,3847 \\
\hline ILMN_16833: & 6482 ST3GAL1 & ST3 beta-galactoside alpha-2,3-sialyltre & 1,5 & 1,5007 \\
\hline ILMN_16851: & 311 ANXA11 & annexin A11 & 1,5 & 0,3847 \\
\hline ILMN_16928: & 64718 UNKL & unkempt homolog (Drosophila)-like & 1,5 & 1,5007 \\
\hline ILMN_173121 & 57714 RNF213 & KIAA1618 & 1,5 & 0,6097 \\
\hline ILMN_17716: & 6629 SNRPB2 & small nuclear ribonucleoprotein polyp $\epsilon$ & 1,5 & 1,8141 \\
\hline ILMN_16997| & 372 ARCN1 & archain 1 & 1,5 & 1,8141 \\
\hline ILMN_23630: & 5888 RAD51 & RAD51 homolog (S. cerevisiae) & 1,5 & 0,3847 \\
\hline ILMN_23655، & 7862 BRPF1 & bromodomain and PHD finger containi & 1,5 & 0,3847 \\
\hline ILMN_18029| & 51588 PIAS4 & protein inhibitor of activated STAT, 4 & 1,5 & 0,3847 \\
\hline ILMN_21847i & 150274 HSCB & HscB iron-sulfur cluster co-chaperone $r$ & 1,5 & 1,0331 \\
\hline ILMN_16993i & 84820 POLR2J4 & polymerase (RNA) II (DNA directed) pol & 1,5 & 0,0000 \\
\hline ILMN_16525: & 29797 DKFZp434K1 & POM121 transmembrane nucleoporin- & 1,5 & 0,7969 \\
\hline ILMN_173981 & 54820 NDE1 & nudE nuclear distribution E homolog 1 & 1,5 & 0,7969 \\
\hline ILMN_33044: & 728640 LOC728640 & family with sequence similarity $133, \mathrm{~m}$ & 1,5 & 0,3847 \\
\hline ILMN_22054: & 202134 FAM153B & family with sequence similarity $153, \mathrm{~m} \bullet$ & 1,5 & 2,0799 \\
\hline ILMN_17676! & 25851 TECPR1 & tectonin beta-propeller repeat contain & 1,5 & 0,7969 \\
\hline ILMN_17757| & 55720 TSR1 & TSR1, 20S rRNA accumulation, homolo & 1,5 & 0,7969 \\
\hline ILMN_18119! & 27246 ZNF364 & ring finger protein 115 & 1,5 & 0,7969 \\
\hline ILMN_169881 & 51776 ZAK & sterile alpha motif and leucine zipper c & 1,5 & 2,0799 \\
\hline ILMN_18027! & 79647 AKIRIN1 & akirin 1 & 1,5 & 1,8141 \\
\hline ILMN_21304: & 10945 KDELR1 & KDEL (Lys-Asp-Glu-Leu) endoplasmic re & 1,5 & 1,5007 \\
\hline ILMN_17087: & 55766 H2AFJ & H2A histone family, member J & 1,5 & 1,5007 \\
\hline ILMN_17222: & 5048 PAFAH1B1 & platelet-activating factor acetylhydrola & 1,5 & 2,0799 \\
\hline ILMN_16815! & 5770 PTPN1 & protein tyrosine phosphatase, non-rect & 1,5 & 1,0331 \\
\hline ILMN_16902: & 84054 PCDHB19P & protocadherin beta 19 pseudogene & 1,5 & 0,3847 \\
\hline ILMN_17200! & 79618 нМВOХ1 & homeobox containing 1 & 1,5 & 1,2499 \\
\hline ILMN_32377" & 55331 ACER3 & alkaline ceramidase 3 & 1,5 & 0,3847 \\
\hline ILMN_17458، & 57238 KIAA0492 & KIAA0492 protein & 1,5 & 2,6244 \\
\hline ILMN_16998! & 5900 RALGDS & ral guanine nucleotide dissociation stin & 1,4 & 0,7969 \\
\hline ILMN_17728 & 55893 ZNF395 & zinc finger protein 395 & 1,4 & 0,7969 \\
\hline ILMN_17369: & 7357 UGCG & UDP-glucose ceramide glucosyltransfer & 1,4 & 2,0799 \\
\hline ILMN_17619! & 6430 SFRS5 & serine/arginine-rich splicing factor 5 & 1,4 & 1,2499 \\
\hline ILMN_16614! & 10471 PFDN6 & prefoldin subunit 6 & 1,4 & 1,0331 \\
\hline ILMN_23944: & 283635 FAM177A1 & family with sequence similarity $177, \mathrm{~m}$ & 1,4 & 2,0799 \\
\hline ILMN_214321 & 541578 CXorf40B & chromosome $X$ open reading frame 40 I & 1,4 & 2,6244 \\
\hline ILMN_16710: & 2591 GALNT3 & UDP-N-acetyl-alpha-D-galactosamine:p & 1,4 & 1,8141 \\
\hline ILMN_17149! & 1629 DBT & dihydrolipoamide branched chain tran! & 1,4 & 1,2499 \\
\hline ILMN_32420: & 493869 GPX8 & glutathione peroxidase 8 (putative) & 1,4 & 1,0331 \\
\hline
\end{tabular}




\begin{tabular}{|c|c|c|c|c|}
\hline ILMN_16982: & 5935 RBM3 & RNA binding motif (RNP1, RRM) proteiı & 1,4 & 1,2499 \\
\hline ILMN_32386: & 93556 C3orf50 & EGF-like and EMI domain containing 1, & 1,4 & 1,8141 \\
\hline ILMN_21019: & 3187 HNRPH1 & heterogeneous nuclear ribonucleoprot & 1,4 & 0,6097 \\
\hline ILMN_16558 & 57146 TMEM159 & transmembrane protein 159 & 1,4 & 2,0799 \\
\hline ILMN_16623i & 9406 ZRANB2 & zinc finger, RAN-binding domain contai & 1,4 & 0,7969 \\
\hline ILMN_20903! & 81875 ISG20L2 & interferon stimulated exonuclease gen & 1,4 & 2,0799 \\
\hline ILMN_17137: & 25 ABL1 & c-abl oncogene 1 , non-receptor tyrosin & 1,4 & 2,0799 \\
\hline ILMN_20729: & 7156 ТОРЗА & topoisomerase (DNA) III alpha & 1,4 & 1,2499 \\
\hline ILMN_209561 & 80008 TMEM156 & transmembrane protein 156 & 1,4 & 0,7969 \\
\hline ILMN_32401: & 84298 LLPH & LLP homolog, long-term synaptic facilit & 1,4 & 1,2499 \\
\hline ILMN_17984! & 23254 KIAA1026 & kazrin, periplakin interacting protein & 1,4 & 0,7969 \\
\hline ILMN_16808! & 5965 RECQL & RecQ protein-like (DNA helicase Q1-lik€ & 1,4 & 0,3847 \\
\hline ILMN_17881i & 5872 RAB13 & RAB13, member RAS oncogene family & 1,4 & 1,5007 \\
\hline ILMN_16784! & 162966 ZNF600 & zinc finger protein 600 & 1,4 & 0,7969 \\
\hline ILMN_16514! & 57222 ERGIC1 & endoplasmic reticulum-golgi intermedi & 1,4 & 0,3847 \\
\hline ILMN_16563! & 5523 PPP2R3A & protein phosphatase 2 , regulatory subı & 1,4 & 1,5007 \\
\hline ILMN_17817! & 2079 ERH & enhancer of rudimentary homolog (Drc & 1,4 & 2,6244 \\
\hline ILMN_21972، & 11128 POLR3A & polymerase (RNA) III (DNA directed) po & 1,4 & 1,5007 \\
\hline ILMN_21234: & 656 ВMP8B & bone morphogenetic protein $8 b$ & 1,4 & 0,3847 \\
\hline ILMN_17628: & 9931 HELZ & helicase with zinc finger & 1,4 & 2,0799 \\
\hline ILMN_16828: & 8209 C21orf33 & open reading frame $3:$ & 1,4 & 1,8141 \\
\hline ILMN_17715! & 6240 RRM1 & ribonucleotide reductase M1 & 1,4 & 1,5007 \\
\hline ILMN_32290: & 728310 LOC728310 & golgin A6 family-like 7, pseudogene & 1,4 & 0,7969 \\
\hline ILMN_17410: & 8396 PIP4K2B & phosphatidylinositol-5-phosphate 4-kir & 1,4 & 1,0331 \\
\hline ILMN_22916: & 117177 RAB3IP & RAB3A interacting protein (rabin3) & 1,3 & 1,2499 \\
\hline ILMN_17624: & 200895 DHFRL1 & dihydrofolate reductase-like 1 & 1,3 & 2,6244 \\
\hline ILMN_17340 & 338328 GPIHBP1 & glycosylphosphatidylinositol anchored & 1,3 & 1,2499 \\
\hline ILMN_21034i & 162967 ZNF320 & zinc finger protein 320 & 1,3 & 0,3847 \\
\hline ILMN_18041، & 222068 TMED4 & transmembrane emp24 protein transp & 1,3 & 1,5007 \\
\hline ILMN_17748: & 55591 VEZT & vezatin, adherens junctions transmemk & 1,3 & 0,7969 \\
\hline ILMN_17924! & 3267 HRB & ArfGAP with FG repeats 1 & 1,3 & 1,8141 \\
\hline ILMN_21814: & 147841 SPC24 & SPC24, NDC80 kinetochore complex co & 1,3 & 0,7969 \\
\hline ILMN_16765i & 10390 CEPT1 & choline/ethanolamine phosphotransfe & 1,3 & 0,3847 \\
\hline ILMN_17984! & 3218 HOXВ8 & homeobox B8 & 1,3 & 2,0799 \\
\hline ILMN_23687: & 387522 TMEM189-U & TMEM189-UBE2V1 readthrough & 1,3 & 2,0799 \\
\hline ILMN_24012! & 10144 FAM13A & family with sequence similarity 13, meı & 1,3 & 1,2499 \\
\hline ILMN_16684، & 10350 АВСА9 & ATP-binding cassette, sub-family $A$ ( $A B$ & 1,3 & 0,7969 \\
\hline ILMN_17710: & 2617 GARS & glycyl-tRNA synthetase & 1,3 & 2,6244 \\
\hline ILMN_17780: & 55066 PDPR & pyruvate dehydrogenase phosphatase & 1,3 & 2,6244 \\
\hline ILMN_32364! & 339736 AK2P2 & adenylate kinase 2 pseudogene 2 & 1,3 & 0,7969 \\
\hline ILMN_18057 & 389677 RBM12B & RNA binding motif protein $12 \mathrm{~B}$ & 1,3 & 0,7969 \\
\hline ILMN_32396! & 84839 RAX2 & retina and anterior neural fold homeok & 1,3 & 0,7969 \\
\hline ILMN_214711 & 440348 LOC440348 & nuclear pore complex interacting prot $€$ & 1,3 & 1,5007 \\
\hline ILMN_17425| & 55149 PAPD1 & mitochondrial poly $(A)$ polymerase & 1,3 & 1,0331 \\
\hline ILMN_20579: & 51101 FAM164A & zinc finger, $\mathrm{C} 2 \mathrm{HC}$-type containing $1 \mathrm{~A}$ & 1,3 & 0,3847 \\
\hline ILMN_236801 & 6942 TCF20 & transcription factor 20 (AR1) & 1,3 & 0,7969 \\
\hline
\end{tabular}




\begin{tabular}{|c|c|c|c|c|}
\hline ILMN_17566: & 116115 ZNF526 & zinc finger protein 526 & 1,3 & 2,6244 \\
\hline ILMN_17910! & 51535 PPHLN1 & periphilin 1 & 1,3 & 1,8141 \\
\hline ILMN_17188! & 3225 HOXC9 & homeobox C9 & 1,3 & 1,8141 \\
\hline ILMN_17310، & 29896 TRA2A & transformer 2 alpha homolog (Drosopr & 1,3 & 1,0331 \\
\hline ILMN_240741 & 10922 FASTK & Fas-activated serine/threonine kinase & 1,3 & 0,3847 \\
\hline ILMN_33081: & 26834 RNU4-2 & RNA, U4 small nuclear 2 & 1,3 & 2,6244 \\
\hline ILMN_17140: & 57563 KLHL8 & kelch-like 8 (Drosophila) & 1,3 & 0,7969 \\
\hline ILMN_323861 & 692205 SNORD89 & small nucleolar RNA, C/D box 89 & 1,3 & 1,2499 \\
\hline ILMN_17196، & 9725 TMEM63A & transmembrane protein $63 \mathrm{~A}$ & 1,3 & 2,0799 \\
\hline ILMN_175341 & 55095 SAMD4B & sterile alpha motif domain containing $\angle$ & 1,3 & 2,6244 \\
\hline ILMN_22596: & 55904 MLL5 & myeloid/lymphoid or mixed-lineage leı & 1,3 & 2,6244 \\
\hline ILMN_17876، & 166647 GPR125 & G protein-coupled receptor 125 & 1,3 & 1,0331 \\
\hline ILMN_174811 & 1642 DDB1 & damage-specific DNA binding protein 1 & 1,3 & 0,7969 \\
\hline ILMN_16921! & 2768 GNA12 & guanine nucleotide binding protein ( $\mathrm{G}$ & 1,3 & 2,6244 \\
\hline ILMN_17304! & 114793 FMNL2 & formin-like 2 & 1,3 & 2,0799 \\
\hline ILMN_171411 & 94241 TP53INP1 & tumor protein p53 inducible nuclear pr & 1,3 & 0,6097 \\
\hline ILMN_173331 & 83939 EIF2A & eukaryotic translation initiation factor : & 1,3 & 1,5007 \\
\hline ILMN_17577: & 734 OSGIN2 & oxidative stress induced growth inhibit & 1,3 & 2,6244 \\
\hline ILMN_21750" & 6429 SFRS4 & serine/arginine-rich splicing factor 4 & 1,3 & 2,6244 \\
\hline ILMN_172471 & 8780 RIOK3 & RIO kinase 3 (yeast) & 1,3 & 0,3847 \\
\hline ILMN_17373! & 4000 LMNA & $\operatorname{lamin} \mathrm{A} / \mathrm{C}$ & 1,3 & 1,5007 \\
\hline ILMN_17656، & 10371 SEMA3A & sema domain, immunoglobulin domair & 1,2 & 2,6244 \\
\hline ILMN_23303" & 128387 TATDN3 & TatD DNase domain containing 3 & 1,2 & 1,2499 \\
\hline ILMN_17669| & 54988 ACSM5 & acyl-CoA synthetase medium-chain fan & 1,2 & 1,2499 \\
\hline ILMN_16768، & 9975 NR1D2 & nuclear receptor subfamily 1 , group D, & 1,2 & 2,0799 \\
\hline ILMN_23228، & 51535 PPHLN1 & periphilin 1 & 1,2 & 2,6244 \\
\hline ILMN_16834" & 10452 TOMM40 & translocase of outer mitochondrial meı & 1,2 & 1,5007 \\
\hline ILMN_16914i & 83752 LONP2 & Ion peptidase 2 , peroxisomal & 1,2 & 1,5007 \\
\hline ILMN_32470: & 26781 SNORA67 & small nucleolar RNA, H/ACA box 67 & 1,2 & 2,6244 \\
\hline ILMN_16735، & 127428 C1orf83 & transcription elongation factor $\mathrm{A}(\mathrm{SII}) \mathrm{N}$ & 1,2 & 2,0799 \\
\hline ILMN_17760: & 165324 UBXN2A & UBX domain protein $2 \mathrm{~A}$ & 1,2 & 1,2499 \\
\hline ILMN_21184" & 84293 C10orf58 & family with sequence similarity $213, \mathrm{~m} \iota$ & 1,2 & 0,3847 \\
\hline ILMN_32419" & 246721 POLR2J2 & polymerase (RNA) II (DNA directed) pol & 1,2 & 1,2499 \\
\hline ILMN_32491: & 81566 CSRNP2 & cysteine-serine-rich nuclear protein 2 & 1,2 & 1,5007 \\
\hline ILMN_17927: & 1613 DAPK3 & death-associated protein kinase 3 & 1,2 & 2,6244 \\
\hline ILMN_16598i & 26472 PPP1R14B & protein phosphatase 1 , regulatory (inhi & 1,2 & 1,8141 \\
\hline ILMN_17271: & 284390 ZNF763 & zinc finger protein 763 & 1,2 & 0,3847 \\
\hline ILMN_22909! & 9973 CCS & copper chaperone for superoxide dism & 1,2 & 1,0331 \\
\hline ILMN_21898 & 51077 FCF1 & FCF1 small subunit (SSU) processome c & 1,2 & 2,0799 \\
\hline ILMN_17687، & 81608 FIP1L1 & FIP1 like 1 (S. cerevisiae) & 1,2 & 1,2499 \\
\hline ILMN_23121، & 90338 ZNF160 & zinc finger protein 160 & 1,2 & 0,3847 \\
\hline ILMN_171021 & 79750 ZNF385D & zinc finger protein 385D & 1,2 & 0,7969 \\
\hline ILMN_171031 & 644591 LOC644591 & peptidylprolyl isomerase A (cyclophilin & 1,2 & 0,3847 \\
\hline ILMN_16827: & 8870 IER3 & immediate early response 3 & 1,2 & 0,7969 \\
\hline ILMN_21499! & 400511 FLJ45256 & uncharacterized LOC400511 & 1,2 & 0,3847 \\
\hline ILMN_16905، & 5533 РРP3СС & protein phosphatase 3 , catalytic subun & 1,2 & 1,0331 \\
\hline
\end{tabular}




\begin{tabular}{|c|c|c|c|c|}
\hline ILMN_18151: & 400931 FLJ27365 & MIRLET7B host gene (non-protein codil & 1,2 & 1,2499 \\
\hline ILMN_17924i & 10109 ARPC2 & actin related protein $2 / 3$ complex, subı & 1,2 & 2,6244 \\
\hline ILMN_17373، & 96459 FNIP1 & folliculin interacting protein 1 & 1,2 & 1,8141 \\
\hline ILMN_17116: & 6477 SIAH1 & siah E3 ubiquitin protein ligase 1 & 1,2 & 0,7969 \\
\hline ILMN_16954I & 5786 PTPRA & protein tyrosine phosphatase, receptoı & 1,2 & 1,0331 \\
\hline ILMN_17634: & 23064 SETX & senataxin & 1,2 & 0,3847 \\
\hline ILMN_172461 & 29988 SLC2A8 & solute carrier family 2 (facilitated gluco & 1,2 & 1,2499 \\
\hline ILMN_22290: & 10201 NME6 & NME/NM23 nucleoside diphosphate ki & 1,2 & 0,3847 \\
\hline ILMN_16633! & 991 CDC20 & cell division cycle 20 homolog (S. cerev & 1,2 & 1,8141 \\
\hline ILMN_17194: & 4437 MSH3 & mutS homolog 3 (E. coli) & 1,2 & 0,3847 \\
\hline ILMN_16559: & 22936 ELL2 & elongation factor, RNA polymerase II, 2 & 1,2 & 1,2499 \\
\hline ILMN_17138! & 201895 C4orf34 & chromosome 4 open reading frame 34 & 1,2 & 0,3847 \\
\hline ILMN_17756! & 8672 EIF4G3 & eukaryotic translation initiation factor ، & 1,2 & 1,2499 \\
\hline ILMN_32489، & 619208 C6orf225 & chromosome 6 open reading frame 22! & 1,2 & 1,5007 \\
\hline ILMN_17227: & 9743 RICS & Rho GTPase activating protein 32 & 1,2 & 1,5007 \\
\hline ILMN_17726! & 23405 DICER1 & dicer 1 , ribonuclease type III & 1,2 & 1,0331 \\
\hline ILMN_18009! & 5717 PSMD11 & proteasome (prosome, macropain) 26s & 1,2 & 0,3847 \\
\hline ILMN_170851 & 27125 AFF4 & AF4/FMR2 family, member 4 & 1,2 & 2,0799 \\
\hline ILMN_177551 & 1540 CYLD & cylindromatosis (turban tumor syndror & 1,2 & 0,7969 \\
\hline ILMN_22156، & 113457 TUBA3D & tubulin, alpha & 1,2 & 2,0799 \\
\hline ILMN_22835! & 54463 FAM134B & family with sequence similarity $134, \mathrm{~m}$ & 1,2 & 1,5007 \\
\hline ILMN_17284: & 10565 ARFGEF1 & ADP-ribosylation factor guanine nuclec & 1,2 & 0,7969 \\
\hline ILMN_17011: & 9424 KCNK6 & potassium channel, subfamily $\mathrm{K}$, memb & 1,2 & 0,7969 \\
\hline ILMN_17920! & $55596 \mathrm{ZCCHC} 8$ & zinc finger, $\mathrm{CCHC}$ domain containing 8 & 1,2 & 2,0799 \\
\hline ILMN_16808! & 10046 MAMLD1 & mastermind-like domain containing 1 & 1,2 & 0,3847 \\
\hline ILMN_17787i & 51421 AMOTL2 & angiomotin like 2 & 1,2 & 0,7969 \\
\hline ILMN_17010! & 2055 CLN8 & ceroid-lipofuscinosis, neuronal 8 (epile & 1,2 & 1,0331 \\
\hline ILMN_20986، & 3995 FADS3 & fatty acid desaturase 3 & 1,2 & 2,0799 \\
\hline ILMN_32315! & 91584 PLXNA4 & plexin A4 & 1,2 & 0,7969 \\
\hline ILMN_17225: & 905 CCNT2 & cyclin T2 & 1,1 & 2,0799 \\
\hline ILMN_219711 & 55308 DDX19A & DEAD (Asp-Glu-Ala-Asp) box polypeptic & 1,1 & 0,6097 \\
\hline ILMN_16739: & 2969 GTF2I & general transcription factor Ili & 1,1 & 2,0799 \\
\hline ILMN_16616I & 401475 SRRM1L & serine/arginine repetitive matrix 1 pseı & 1,1 & 1,5007 \\
\hline ILMN_17431: & 23215 BAT2D1 & proline-rich coiled-coil $2 \mathrm{C}$ & 1,1 & 2,0799 \\
\hline ILMN_17912: & 85369 FAM40A & family with sequence similarity 40, meı & 1,1 & 2,6244 \\
\hline ILMN_175311 & 65125 WNK1 & WNK lysine deficient protein kinase 1 & 1,1 & 2,6244 \\
\hline ILMN_17827، & 90333 ZNF468 & zinc finger protein 468 & 1,1 & 0,3847 \\
\hline ILMN_17548: & 81545 FBXO38 & F-box protein 38 & 1,1 & 1,0331 \\
\hline ILMN_17429: & 7582 ZNF33B & zinc finger protein 33B & 1,1 & 2,0799 \\
\hline ILMN_17348: & 4524 MTHFR & methylenetetrahydrofolate reductase 1 & 1,1 & 1,0331 \\
\hline ILMN_17929: & $4335 \mathrm{MNT}$ & MAX binding protein & 1,1 & 1,8141 \\
\hline ILMN_17202، & 9320 TRIP12 & thyroid hormone receptor interactor 1 : & 1,1 & 2,6244 \\
\hline ILMN_16685! & 11037 STON1 & stonin 1 & 1,1 & 0,7969 \\
\hline ILMN_17251i & 6905 TBCE & tubulin folding cofactor $E$ & 1,1 & 1,0331 \\
\hline ILMN_16537: & 130617 ZFAND2B & zinc finger, AN1-type domain 2B & 1,1 & 1,2499 \\
\hline
\end{tabular}


